\title{
Down-regulation of microRNA-144-3p and its clinical value in non-small cell lung cancer: a comprehensive analysis based on microarray, miRNA-sequencing, and quantitative real-time PCR data
}

Yu-Ji Chen ${ }^{1 \dagger}$, Yi-Nan Guo ${ }^{2+}$, Ke Shi $^{3}$, Hui-Mei Huang ${ }^{1}$, Shu-Ping Huang ${ }^{1}$, Wen-Qing Xu' ${ }^{1}, Z u-Y u n ~ L i{ }^{3}$, Kang-Lai Wei ${ }^{2^{*}}$, Ting-Qing Gan ${ }^{1 *}$ and Gang Chen ${ }^{3}$

\begin{abstract}
Background: Previous studies have shown that miR-144-3p might be a potential biomarker in non-small cell lung cancer (NSCLC). Nevertheless, the comprehensive mechanism behind the effects of miR-144-3p on the origin, differentiation, and apoptosis of NSCLC, as well as the relationship between miR-144-3p and clinical parameters, has been rarely reported.

Methods: We investigated the correlations between miR-144-3p expression and clinical characteristics through data collected from Gene Expression Omnibus (GEO) microarrays, the relevant literature, The Cancer Genome Atlas (TCGA), and real-time quantitative real-time PCR (RT-qPCR) analyses to determine the clinical role of miR-144-3p in NSCLC. Furthermore, we investigated the biological function of miR-144-3p by Gene Ontology (GO), Kyoto Encyclopedia of Genes and Genomes (KEGG) analyses. Protein-protein interaction (PPI) network was created to identify the hub genes.

Results: From the comprehensive meta-analysis, the combined SMD of miR-144-3p was -0.95 with $95 \% \mathrm{Cl}$ of $(-1.37,-0.52)$, indicating that less miR-144-3p was expressed in the NSCLC tissue than in the normal tissue. MiR-144-3p expression was significantly correlated with stage, lymph node metastasis and vascular invasion (all $P<0.05)$. As for the bioinformatics analyses, a total of 37 genes were chosen as the potential targets of miR144-3p in NSCLC. These promising target genes were highly enriched in various key pathways such as the protein digestion and absorption and the thyroid hormone signaling pathways. Additionally, PPI revealed five genes-C12orf5, CEP55, E2F8, STIL, and TOP2A—as hub genes with the threshold value of 6.
\end{abstract}

Conclusions: The current study validated that miR-144-3p was lowly expressed in NSCLC. More importantly, miR-144-3p might function as a latent tumor biomarker in the prognosis prediction for NSCLC. The results of bioinformatics analyses may present a new method for investigating the pathogenesis of NSCLC.

Keywords: MiR-144-3p, Non-small cell lung cancer, Microarray, miRNA-sequencing, Quantitative real-time PCR

\footnotetext{
* Correspondence: yxwwkl@163.com; gantingqing_gxmu@163.com

${ }^{\dagger} \mathrm{Yu}$-Ji Chen and Yi-Nan Guo contributed equally to this work.

²Department of Pathology, Second Affiliated Hospital of Guangxi Medical

University, Daxuedong Road, Nanning, Guangxi Zhuang Autonomous Region

530021, People's Republic of China

${ }^{1}$ Department of Medical Oncology, Second Affiliated Hospital of Guangxi

Medical University, Daxuedong Road, Nanning, Guangxi Zhuang

Autonomous Region 530021, People's Republic of China

Full list of author information is available at the end of the article
}

(c) The Author(s). 2019 Open Access This article is distributed under the terms of the Creative Commons Attribution 4.0 International License (http://creativecommons.org/licenses/by/4.0/), which permits unrestricted use, distribution, and reproduction in any medium, provided you give appropriate credit to the original author(s) and the source, provide a link to the Creative Commons license, and indicate if changes were made. The Creative Commons Public Domain Dedication waiver (http://creativecommons.org/publicdomain/zero/1.0/) applies to the data made available in this article, unless otherwise stated. 


\section{Introduction}

Lung cancer (LC) is recognized as a life-threatening malady as the incidence and mortality rates are ranked second among all neoplasms worldwide [1]. Accounting for $85 \%$ of all diagnosed LC cases, non-small cell lung cancer (NSCLC) is divided mainly into lung squamous cell carcinoma (LUSC), lung adenocarcinoma (LUAD), and large cell carcinoma (LCC) [2]. Currently, the main therapy for NSCLC is a combination of surgery and chemotherapy [3]. Although great progress has been made in the early detection, diagnosis, and targeted treatments of NSCLC, the five-year survival rates are still low, varying from 4 to $17 \%$ depending on regional differences and the disease stage [3-5]. Patients with severer tumor and comorbidity burdens are at a higher risk of death from not receiving effective or specific treatments [6]. Thus, the understanding of the molecular mechanisms in NSCLC and the identification of new therapeutic targets are crucial.

MiRNAs are single-stranded ncRNAs of approximately 20 nucleotides in length. They play an important role in the regulation of gene expression by post-transcriptionally binding with the mRNAs of target genes [7]. Participating in cellular differentiation and homeostasis, miRNAs play pivotal roles in cancer [8]. Tissue-specific miRNAs act as novel potential biomarkers in the diagnosis, treatment, and prognosis of cancer [9, 10]. Recently, the effects of miRNAs on oncogenesis and tumor progression have been receiving a great deal of attention.

MicroRNA-144-3p (miR-144-3p), having various functions in different types of cancers, is one of the miRNAs related to cancer. MiR-144-3p acts as a suppressive factor in laryngeal squamous cell carcinoma [11], gastric cancer [12], hepatocellular carcinoma [13], and pancreatic cancer [14]. However, it is an oncogene in renal carcinoma [15], nasopharyngeal carcinoma [16], and colorectal cancer [17]. Previous studies have also implicated that miR-144-3p is involved in cell proliferation, apoptosis, and autophagy by targeting the TP53-inducible glycolysis and apoptosis regulator (TIGAR) in LC [18]. The down-regulation of miR144-3p results in metabolic alterations of LC cells by regulating the glucose transporter 1 (GLUT1) [19]. Previous studies have shown that miR-144-3p might be a biomarker and target with great potential. Nevertheless, the comprehensive mechanism behind the effects of miR-144-3p on the origin, differentiation, and apoptosis of NSCLC, as well as the relationship between miR-144-3p and clinical parameters, has been rarely reported.

This study investigated the correlations between miR-144-3p expression and clinical characteristics through data collected from Gene Expression Omnibus (GEO) microarrays, the relevant literature, The Cancer Genome Atlas (TCGA), and real-time quantitative real-time PCR (RT-qPCR) analyses to determine the clinical role of
miR-144-3p in NSCLC. The latent mechanism of action in NSCLC was subsequently examined by using 12 predictive programs to forecast the genes targeted by miR-144-3p. In addition, bioinformatics analyses, which included Gene Ontology (GO), Kyoto Encyclopedia of Genes and Genomes (KEGG), and protein-protein interaction (PPI) network analyses, were performed.

\section{Materials and methods Data collection}

A microarray search of miR-144-3p in NSCLC was conducted in the GEO database (http://www.ncbi.nlm.ni h.gov/geo/) with the following keywords: (MicroRNA OR "Micro RNA" OR "non-coding RNA" OR ncRNA OR "small RNA" OR miRNA) AND (Lung OR pulmonary) AND (cancer OR tumor OR neoplasm OR malignancy OR carcinoma OR adenocarcinoma OR AC OR SCC OR NSCLC). The entry type was restricted to "series," and the organism was filtered by "Homo sapiens." The criteria for inclusion were as follows: (1) patients diagnosed with NSCLC and its subtypes were investigated; (2) cancerous and noncancerous samples were involved; (3) the healthy and malignant groups included at least three samples in the form of tissue, blood, or plasma; (4) and the expression profiling data for miR-144-3p were available. Related studies were retrieved from the PubMed, Google Scholar, China National Knowledge Infrastructure (CNKI), Chongqing VIP electronic (VIP) and Chinese Wanfang databases. Figure 1 shows the workflow for the study.

\section{MicroRNA-144-3p expression data from the Cancer genome atlas}

TCGA (https://cancergenome.nih.gov/) was used for obtaining detailed information about the expression value of miR-144-3p in NSCLC and noncancerous samples. The differences in the miR-144-3p expression in the NSCLC samples and the normal controls were calculated with IBM SPSS Statistics V22.0 software.

\section{Quantitative real-time PCR}

For the current study, 125 matched samples were provided by the Department of Pathology of the First Affiliated Hospital of Guangxi Medical University. Formalin fixation and paraffin embedding (FFPE) were performed to preserve the specimens. This aspect of the research was authorized by the hospital's ethics committee. Next, the miR-144-3p expression in 125 paired clinical samples was detected by RT-qPCR with the Applied Biosystems 7900HT Fast Real-Time PCR System software. The miR-144-3p sequence was as follows: UACAGUAUA GAUGAUGUACU. The formula for $2-\Delta \mathrm{cq}$ was used for the calculation of the miR-144-3p expression value. 


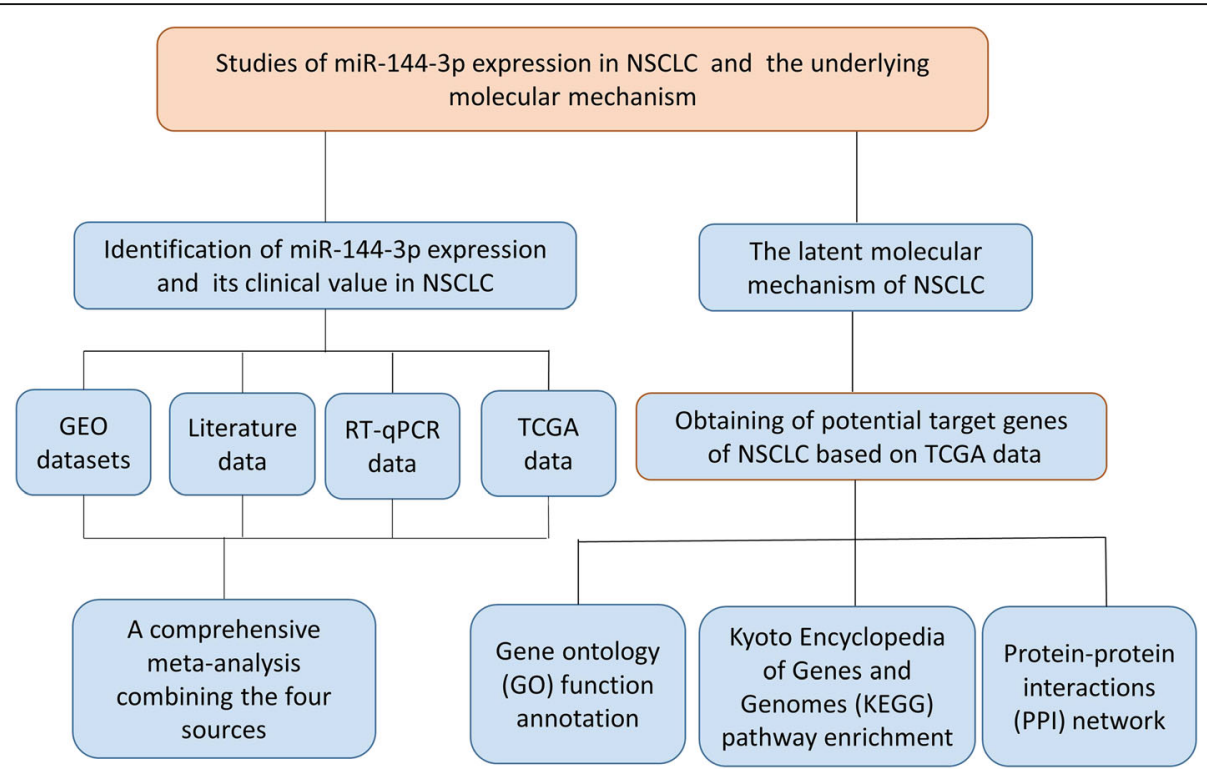

Fig. 1 The flow diagram of this study. Note: The workflow indicates that a comprehensive meta-analysis was performed to confirm microRNA144-3p expression in non-small cell lung cancer and that a bioinformatics analysis was conducted to investigate the latent molecular mechanism

\section{Statistical analysis and comprehensive meta-analysis}

After miR-144-3p was $\log _{2}$-transformed, the expression profiling information was used to calculate the number, mean (M) and standard deviation (SD) for each control and experimental group with IBM SPSS Statistics V22.0 software. In addition, Stata 12.0 software was used for performing a comprehensive meta-analysis of data aggregated from multiple sources (microarray, literature, miRNA sequencing, and RT-qPCR). The analysis of the miR-144-3p expression in the NSCLC and tumor-free specimens was displayed on forest plots that illustrate the standardized mean difference (SMD) and the 95\% confidential interval (CI). The chi-squared test of $\mathrm{Q}$ and the $\mathrm{I}^{2}$ statistic were calculated to assess heterogeneity across the studies and to determine the appropriateness of applying either a random effects model or fixed effects model to the pooling process. To measure publication bias, Egger's and Begg's tests and a funnel plot, for which significance was $p<0.05$, were performed.

\section{Latent targets of microRNA-144-3p in non-small cell lung} cancer

MiRWALK2.0, an online archive of data on miRNA-target interactions [20], was mined to forecast the genes targeted by miR-144-3p. In total, 12 servers with miRWalk, miRMap, MicroT4, miRNAMap, TargetScan PICTAR2, miRBridge, PITA, miRanda, RNAhybrid, miRDB, RNA22 were used. Only those genes projected by more than six of the servers were recognized as target genes. The high-expressed genes in LUAD and LUSC were acquired through Gene Expression Profiling Interactive Analysis (GEPIA). The overlapping genes among the up-regulated genes in LUAD and LUSC and the predicted target genes, were viewed as promising targets of miR144-3p in NSCLC. A review of the literature on the specific target genes of miR-144-3p in NSCLC was conducted. The target genes determined by previous studies and the predicted target genes, namely promising target genes, were used in the functional analysis.

\section{Functional analysis for promising target genes}

The GO vocabularies, which include biological processes (BPs), cellular components (CCs), and molecular functions (MFs), were enriched by Metascape (http://metascape.org/ $\mathrm{gp} /$ ). The functional annotation of the underlying target genes was then elucidated by KEGG pathway analysis with Metascape tool. In addition, a PPI network was constructed to reveal the hub genes of the potential target genes on STRING, a web portal for undermining the integrated function of multiple genes [21].

\section{Expression of hub genes from the Cancer genome atlas and the genotype-tissue expression database}

To further confirm the function of hub genes in NSCLC and their relationship to miR-144-3p, a search of TCGA and the Genotype-Tissue Expression (GTEx) database was performed to determine the expression pattern of the hub genes in NSCLC. Box plots of the hub genes in the NSCLC and non-cancer samples were developed through GEPIA. 


\section{Results}

Confirmation of the expression and clinical value of microRNA-144-3p in non-small cell lung cancer, based on gene expression omnibus datasets

MicroRNA-144-3p expression in non-small cell lung cancer obtained through gene expression omnibus microarrays

A total of 19 microarrays from the GEO database met the entry criteria. The features of the included GEO datasets are depicted in Table 1. Of the microarrays, 14 were obtained from tissue, and 5 were derived from blood (GSE27486, GSE40738, GSE64951, GSE93300, and GSE114711). In addition, the expression data from the NSCLC and control groups were collected on the basis of the GEO database. With respect to the data from the tissue samples, the NSCLC groups had a significantly lower level of miR-144-3p expression than the control groups in GSE25508, GSE48414, GSE51853, GSE56036, GSE63805, GSE72526, GSE74190, and GSE102286 $(p=0.0202, p<0.0001, p<0.0001, p=0.0011$, $p<0.0001, p=0.0102, p<0.0001$, and $p<0.0001$, respectively (Fig. 2)). In contrast, no notable distinction in miR-144-3p expression was detected between the NSCLC and the control groups in the other microarrays (GSE14936, GSE29248, GSE36681, GSE47525, GSE53882, and GSE77380). Regarding the data from the blood samples, miR-144-3p expression in NSCLC was found to decrease significantly in GSE27486 and GSE40738 $(p=0.0196, p=0.0036$, respectively (Fig. 3)).

\section{Results of meta-analysis of gene expression omnibus datasets}

A meta-analysis was conducted on the basis of the 19 included microarrays from the GEO database. The results are demonstrated in Fig. 4a. Given the apparent heterogeneity $\left(p<0.05, \mathrm{I}^{2}=94.3 \%\right)$, a random effects model was applied, and remarkable down-regulation $(\mathrm{SMD}=-0.89$; 95\% CI $-1.34,-0.44 ; p=0.000$ ) of miR-144-3p was found in the NSCLC groups. A sensitivity analysis was later conducted to explore whether a particular microarray played a vital role in significant heterogeneity (Fig. 4b). After an individual study was removed each time of meta-analysis, the combined effect was compared to the previous one. No study was found to have played a crucial role in any of the enrolled studies.

A funnel plot was generated to estimate publication bias (Fig. 4c). To further clarify the heterogeneity source, a subgroup analysis was performed. It was based on multiple characteristics: sample source (tissue vs. blood), and cancer type (adenocarcinoma vs. squamous cell carcinoma). As is illustrated in Fig. 5, significant heterogeneity was observed in the tissue subgroup $\left(\mathrm{I}^{2}=95.8 \%\right.$, $p=0.000$ ). Significant heterogeneity was also found in adenocarcinoma $\left(\mathrm{I}^{2}=92.9 \%, p=0.000\right)$ and squamous

Table 1 Features of the enrolled Gene Expression Omnibus datasets

\begin{tabular}{|c|c|c|c|c|c|c|c|c|c|}
\hline \multirow[t]{2}{*}{ Accession } & \multirow[t]{2}{*}{ GPL } & \multirow[t]{2}{*}{ Year } & \multicolumn{3}{|c|}{ NSCLC } & \multicolumn{3}{|c|}{ Control } & \multirow[t]{2}{*}{ Source } \\
\hline & & & $n$ & $M$ & SD & $n$ & $M$ & SD & \\
\hline GSE14936 & GPL8879 & 2012 & 22 & 7.8687 & 0.78175 & 19 & 8.0904 & 0.82617 & tissue \\
\hline GSE25508 & GPL7731 & 2014 & 24 & 7.4846 & 1.24999 & 24 & 8.463 & 1.55049 & tissue \\
\hline GSE27486 & GPL11432 & 2012 & 22 & 1.6495 & 0.90141 & 23 & 2.2125 & 0.64072 & blood \\
\hline GSE29248 & GPL8179 & 2014 & 6 & 672.5763 & 701.2225 & 6 & 648.1356 & 587.8154 & tissue \\
\hline GSE36681 & GPL8179 & 2014 & 103 & 9.2339 & 0.66957 & 103 & 9.2783 & 0.62179 & tissue \\
\hline GSE40738 & GPL16016 & 2017 & 82 & -1.5715 & 0.86663 & 59 & -1.1291 & 0.88742 & blood \\
\hline GSE47525 & GPL17222 & 2015 & 14 & 2.4786 & 0.86574 & 14 & 2.5643 & 1.02852 & tissue \\
\hline GSE48414 & GPL16770 & 2015 & 154 & -0.3765 & 2.18784 & 20 & 1.6223 & 0.82035 & tissue \\
\hline GSE51853 & GPL7341 & 2016 & 126 & -6.9614 & 0.60881 & 5 & -1.5464 & 0.63552 & tissue \\
\hline GSE53882 & GPL18130 & 2017 & 397 & 1.6467 & 1.57039 & 151 & 1.8168 & 1.89299 & tissue \\
\hline GSE56036 & GPL15446 & 2017 & 19 & 17.7554 & 7.25245 & 29 & 100.0724 & 121.8101 & tissue \\
\hline GSE63805 & GPL18410 & 2016 & 32 & 8.286 & 0.89384 & 30 & 10.0377 & 1.15923 & tissue \\
\hline GSE64591 & GPL18942 & 2018 & 100 & 4.9474 & 0.08751 & 100 & 4.9618 & 0.09277 & blood \\
\hline GSE72526 & GPL20275 & 2015 & 67 & 7.2239 & 0.91818 & 18 & 6.6111 & 0.6978 & tissue \\
\hline GSE74190 & GPL19622 & 2015 & 72 & 1.3728 & 1.57232 & 44 & 6.016 & 0.85477 & tissue \\
\hline GSE77380 & GPL16770 & 2016 & 3 & 1.8937 & 4.51735 & 12 & 4.2684 & 3.91992 & tissue \\
\hline GSE93300 & GPL21576 & 2017 & 9 & -6.0441 & 0.99527 & 4 & -6.572 & 3.92546 & blood \\
\hline GSE102286 & GPL23871 & 2018 & 91 & 7.763 & 1.77768 & 88 & 9.1063 & 1.43636 & tissue \\
\hline GSE114711 & GPL18573 & 2018 & 19 & 6.9034 & 1.33845 & 7 & 6.9887 & 1.37975 & blood \\
\hline
\end{tabular}


a

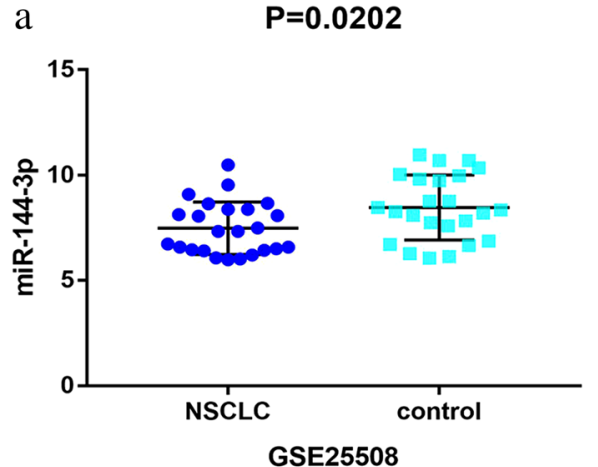

$\mathrm{c}$

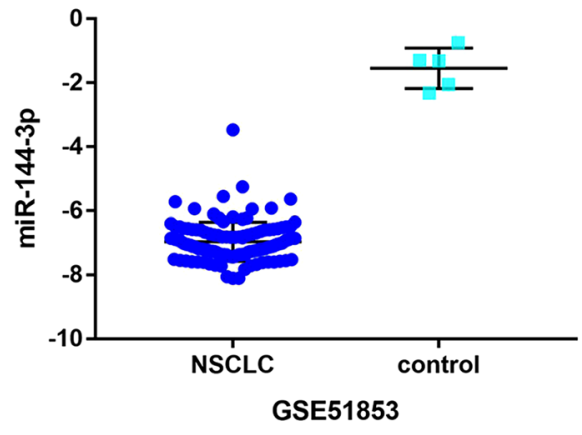

e

$P<0.0001$

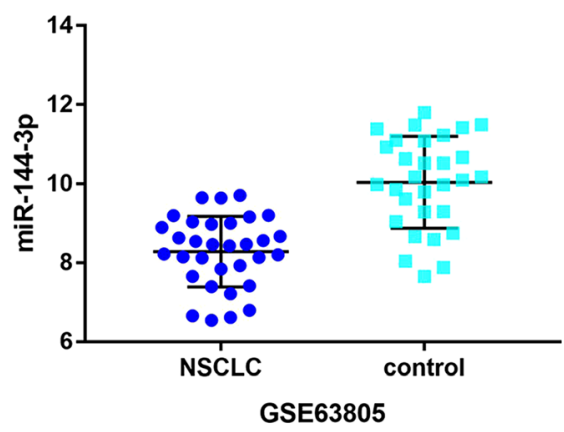

$\mathrm{g}$

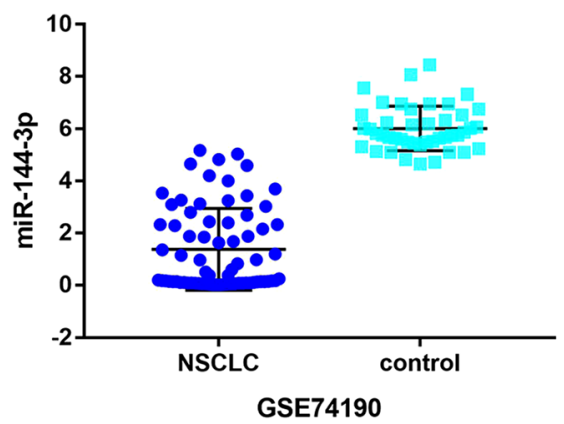

b $P<0.0001$
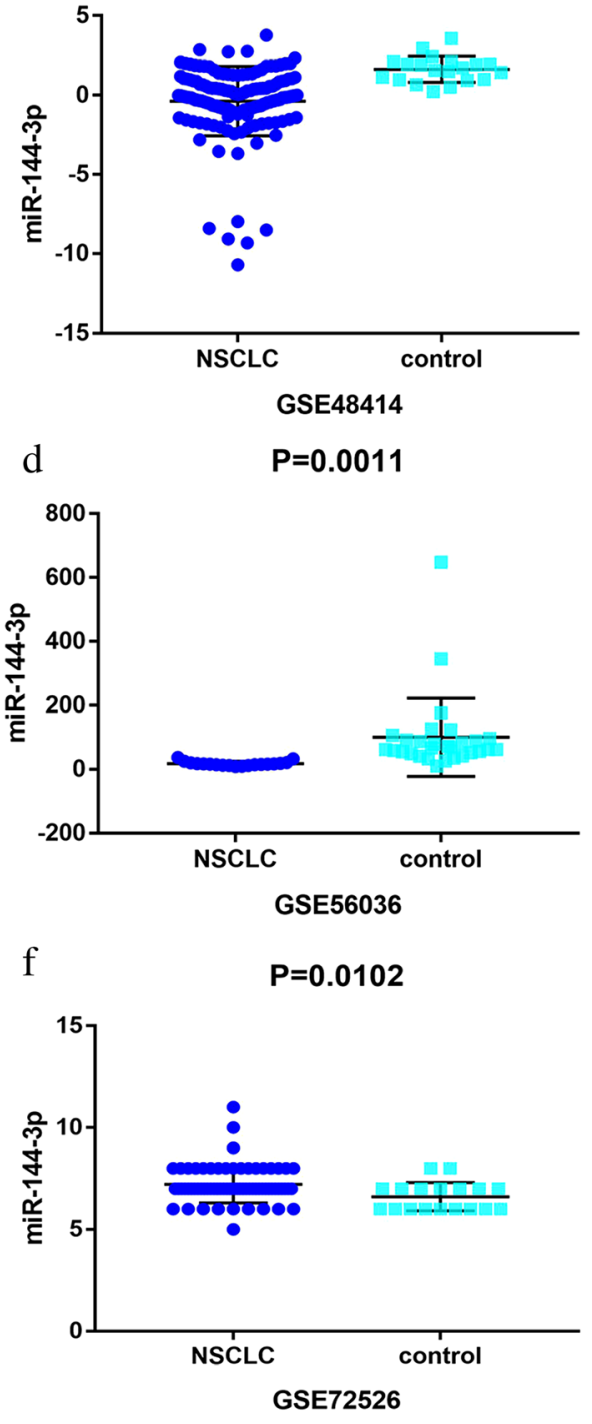

h

$\mathrm{P}<0.0001$

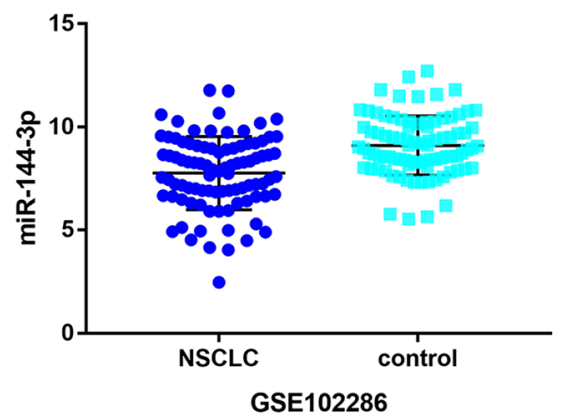

Fig. 2 Down-regulation of microRNA-144-3p in in the other microarrays tissues, based on Gene Expression Omnibus datasets. Notes: a GSE25508. b GSE48414. c GSE51853. d GSE56036. e GSE63805. f GSE72526. g GSE74190. h GSE102286 
a

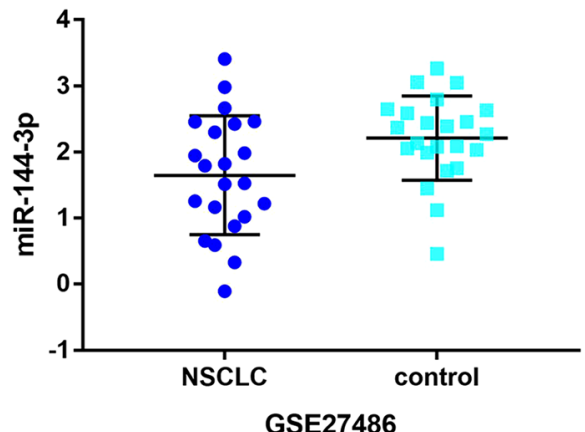

b

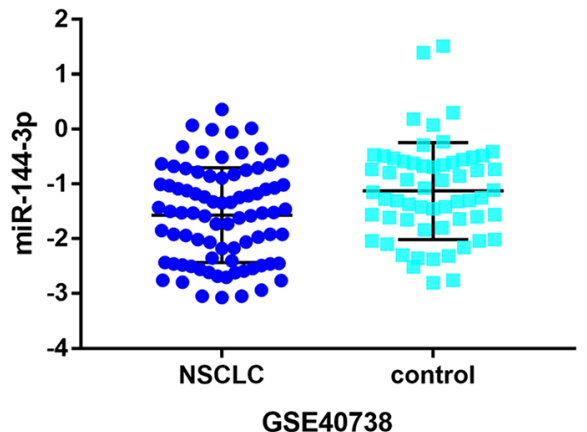

Fig. 3 Low expression of miR-144-3p in non-small cell lung cancer chips derived from blood sample. Notes: a GSE27486. b GSE40738

a Study GSE14936 (2012) GSE25508 (2014) GSE27486 (2012)

GSE29248 (2014) GSE36681 (2014) GSE40738 (2017) GSE47525 (2015) GSE48414 (2015) GSE51853 (2016) GSE53882 (2017) GSE56036 (2017)

GSE63805 (2016) GSE64591 (2018) GSE72526 (2015) GSE74190 (2015)

GSE77380 (2016) GSE93300 (2017) GSE102286 (2018) GSE114711 (2018)

Overall (1-squared $=94.3 \%, p=0.000)$ NOTE: Weights are from random effects analysis

$-10.3$

Begg's funnel plot with pseudo $95 \%$ confidence limits

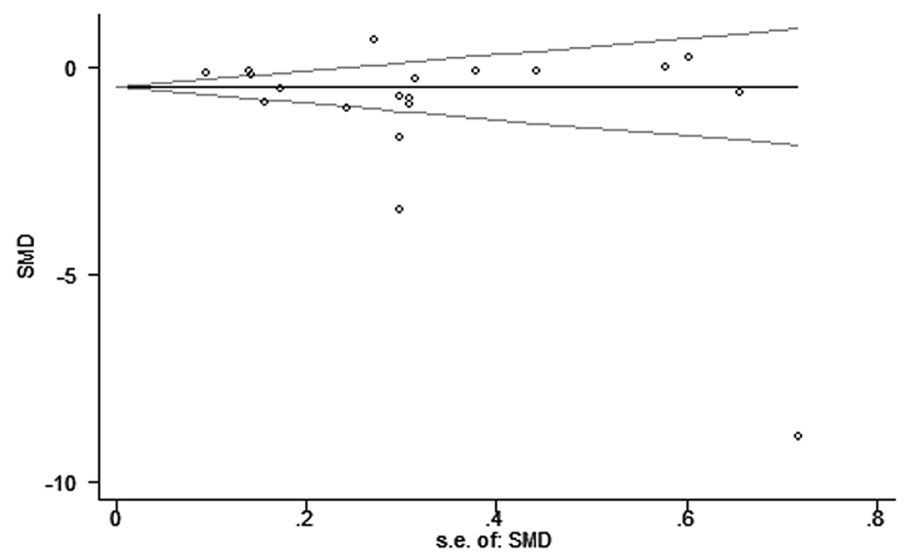
$-0.83(-1.14,-0.52) \quad 5.88$ $-0.06(-0.93,0.80) \quad 4.92$

$-0.89(-1.34,-0.44) \quad 100.00$

$\operatorname{SMD}(95 \% \mathrm{Cl}) \quad$ Weight

$-0.28(-0.89,0.34) \quad 5.42$

$-0.69(-1.28,-0.11) \quad 5.48$

$-0.72(-1.33,-0.12) \quad 5.44$

$0.04(-1.09,1.17) \quad 4.35$

$0.07(-0.34,0.20) \quad 5.91$

$-0.51(-0.85,-0.17) \quad 5.84$

$-0.96(-1.44,-0.48) \quad 5.66$

$8.88(-10.29,-7.48) \quad 3.78$

$-0.10(-0.29,0.09) \quad 5.98$

$-0.87(-1.47,-0.26) \quad 5.44$

$-1.70(-2.28,-1.12) \quad 5.48$

$0.16(-0.44,0.12) \quad 5.91$

$0.70(0.17,1.23)$

$-3.45(-4.03,-2.86)$

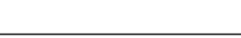

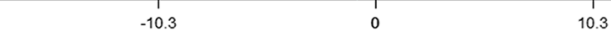

(1)

c

b

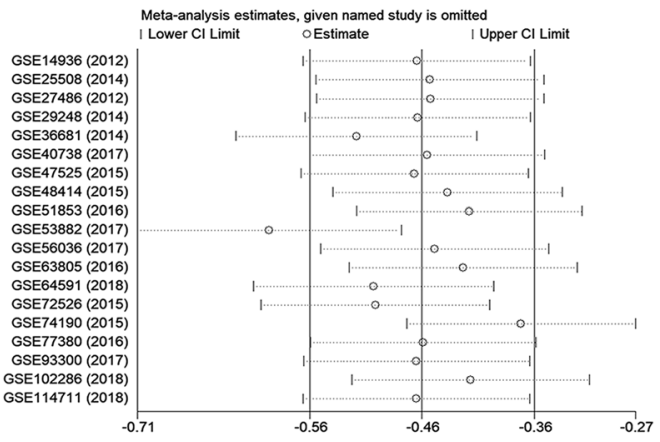

$-0.71$
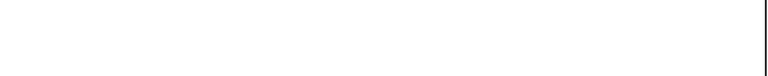

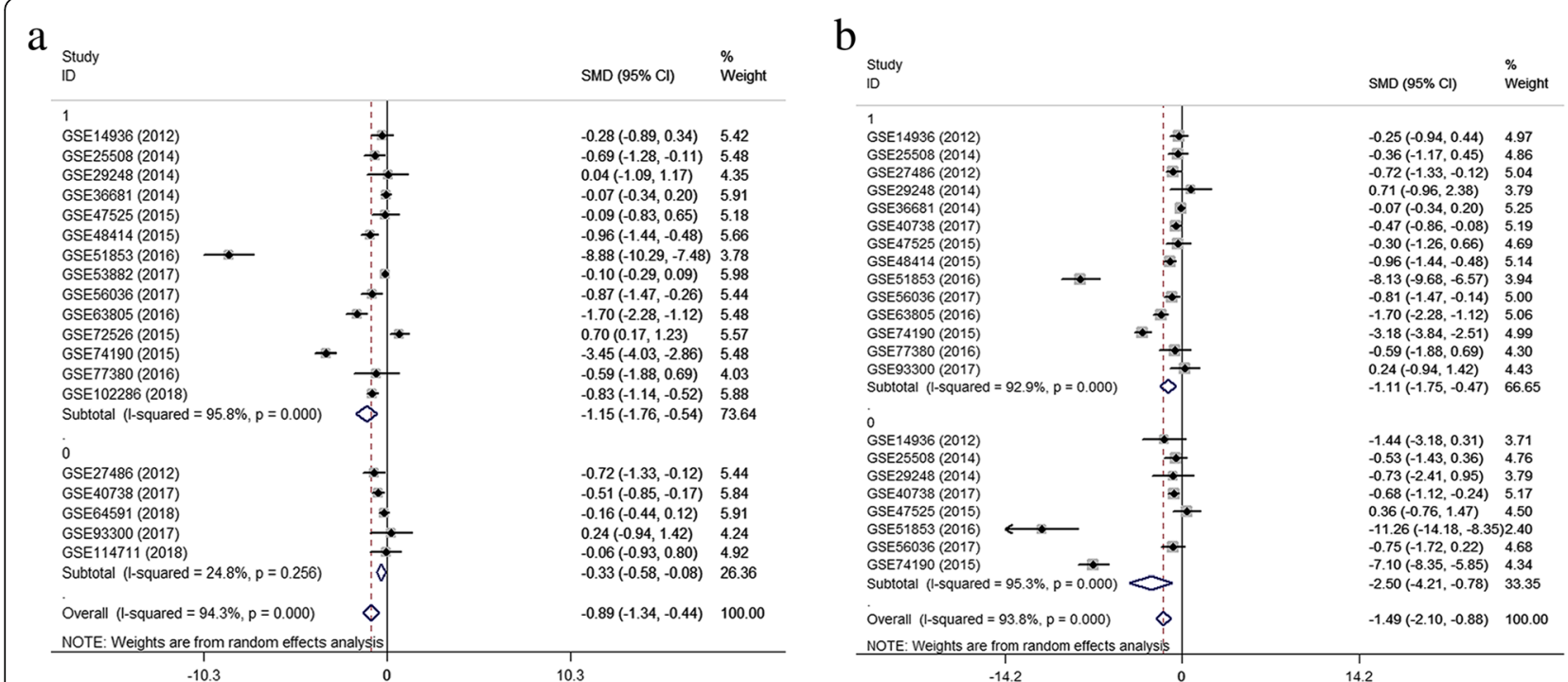

Fig. 5 Results of subgroup analyses. Notes: a Subgroup analysis based on sample source. The tissue subgroup had significant heterogeneity $\left(I^{2}=95.8 \%, p=0.000\right)$. (1: tissue, 0 : blood) $\mathbf{b}$ Subgroup analysis based on cancer type. The adenocarcinoma and the squamous cell carcinoma both had significant heterogeneity (1: adenocarcinoma, 0: squamous cell carcinoma)

cell carcinoma $\left(\mathrm{I}^{2}=95.3 \%, p=0.000\right)$. These results suggest that sample source and cancer type might be sources of heterogeneity.

\section{Literature}

A review of the literature relevant to miR-144-3p was conducted through searches in the PubMed, Google Scholar, CNKI, VIP, and Wanfang databases. Neither the $\mathrm{M}$ nor the SD of miR-144-3p in the NSCLC and normal groups was provided in the literature; therefore, no available data could be obtained from the existing studies.

\section{Confirmation of the expression and clinical effects of microRNA-144-3p in non-small cell lung cancer, based on the Cancer genome atlas data \\ MicroRNA-144-3p expression and prognostic value in non- small cell lung cancer tissues}

TCGA contained 376 samples for LUSC patients and 488 samples for LUAD patients. Regarding LUSC, miR-144-3p expression was remarkably downregulated in comparison with the normal controls (2.8193 \pm 1.40600 vs. $5.5678 \pm 1.27693, p<0.001$ (Fig. 6a and Table 2)). In terms of LUAD, the expression level of miR-144-3p was obviously less than that in the healthy tissue $(2.8959 \pm 1.35967$ vs. $5.2775 \pm 1.64708, p<0.001$ (Fig. $6 \mathrm{~b}$ and Table 3)). The data from LUSC and LUAD were combined for further examination of the miR-144-3p expression in NSCLC. As is illustrated in Fig. 6c and Table 4, miR-144-3p was significantly reduced in the
NSCLC tissue compared to the non-cancerous lung tissue $(2.8632 \pm 1.37928$ vs. $5.4243 \pm 1.4702, p<0.0001)$. A Kaplan-Meier curve was later used to identify the effects of the expression of miR-144-3p on survival time. As is shown in Fig. 7, the $p$ values for the three Kaplan-Meier curves were all greater than 0.05 , thus indicating no significant difference in survival time between the group with low levels of miR-144-3p and the one with high levels.

\section{Relationships between microRNA-144-3p and clinical pathology of non-small cell lung cancer, based on the Cancer genome atlas data}

As can be seen in Tables 2 and 3, the clinical characteristics of 332 LUSC patients and 445 LUAD patients were downloaded from TCGA. Regarding LUSC, a significant difference in miR-144-3p was found for stage $(p=0.040)$ and primary tumor $(\mathrm{T})(p=0.035)$. LUSC patients in Stages III-IV $(2.4469 \pm 1.41079)$ had a lower expression of miR-144-3p than those in Stages I-II (2.8878 \pm 1.39556). The miR-144-3p expression of LUSC in T3T4 $(2.5088 \pm 1.36178)$ was more significantly decreased than T1-T2 $(2.9023 \pm 1.40854)$. In terms of LUAD, a significant difference of miR-144-3p expression was observed for stage $(p=0.031)$. Patients in Stages III-IV $(3.1868 \pm 1.45395)$ had higher expression values of miR-144-3p. The data on LUAD and LUSC, based on TCGA, were pooled for further validation. As is illustrated in Table 4, the significance in the statistics for the $\mathrm{T}$ stage was based on the lower miR-144-3p expression in patients in T3-T4 $(p<0.05)$. 
a

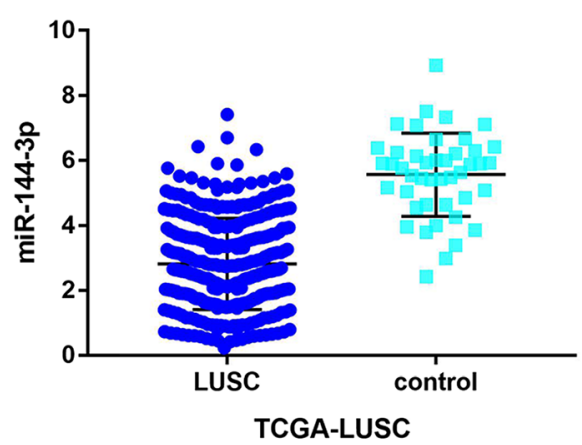

TCGA-LUSC

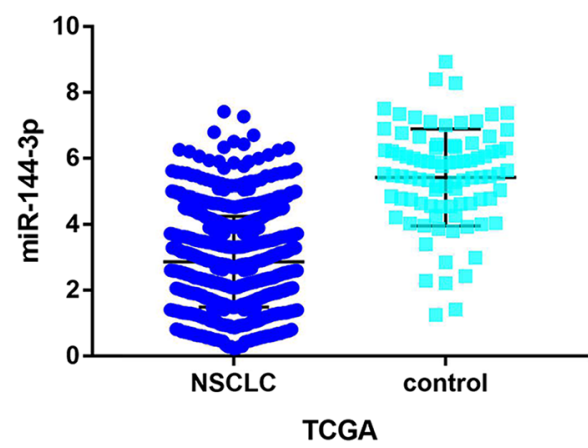

b

$P<0.0001$

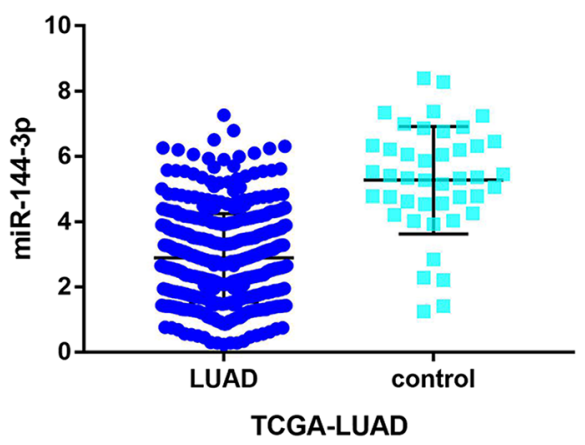

C

$P<0.0001$

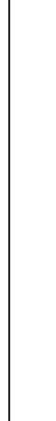

Genome Atlas data. Note: a MicroRNA-144-3p expres
cancer tissues was less than that in normal tissues

\section{Quantitative real-time PCR analysis}

The microRNA-144-3p expression and its significance in non-small cell lung cancer prognoses

Using RT-qPCR, the clinical expression value of miR-144-3p in 125 matched tissues was evaluated. As is illustrated in Fig. 8a and Table 5, the NSCLC samples exhibited a significantly lower expression level of miR-144-3p than the non-cancerous samples $(2.808 \pm 1.303$ vs. $4.813 \pm$ 2.618, $p<0.001)$. Next, miR-144-3p expression was then analyzed for LUAD and for LUSC. As is shown in Fig. 8b and c, unlike what was found in the adjacent non-cancerous tissue, apparently lowly expressed miR-144-3p was observed in both LUSC and LUAD ( $p=0.0004, p<0.0001)$. A Kaplan-Meier curve was generated to assess whether miR-144-3p is appropriate for the prognosis prediction of LUAD. As is depicted in Fig. 9, the LUAD patients who exhibited lower expression values of miR-144-3p might have worse outcomes $(p=0.397)$.

\section{Correlations between microRNA-144-3p expression and} clinical characteristic for non-small cell lung cancer patients The miR-144-3p expression in NSCLC cases was significantly different in lymph node metastasis and vascular invasion (Table 5). The lower value of miR-144-3p was found in patients with lymph node metastasis but not in those without it (Table 5). Patients with vascular invasion maintained a miR-144-3p value of $2.1400 \pm 1.2263$, and the expression level of those without vascular invasion was $3.0682 \pm 1.24395$ (Table 5). To further validate the correlation of miR-144-3p and clinicopathological characteristics, the NSCLC cases were divided into LUSC and LUAD groups. For the LUSC group (Table 6), no statistical differences were seen for smoking, vascular invasion, or lymph node metastasis. However, for LUAD, the statistical analyses indicated significant differences for smoking, vascular invasion, and lymph node metastasis. In comparison to patients without vascular invasion, those with vascular invasion had lower miR-144-3p expression values. The miR-144-3p expression in patients with a smoking habit was significantly down-regulated over that of patients without the habit ( $p=0.027$, Table 7$)$. Besides, the miR-144-3p expression in NSCLC patients who were considered to have lymph node metastasis was markedly reduced.

\section{Meta-analysis of combination of gene expression omnibus, the Cancer genome atlas, and quantitative real-time PCR data}

Because no relevant studies were found, data from three sources (microarrays, miRNA-sequencing, and RT-qPCR) containing 2264 NSCLC samples and 968 non-cancerous 
Table 2 Association between microRNA-144-3p expression and clinicopathological parameters in lung squamous cell carcinoma, based on The Cancer Genome Atlas data

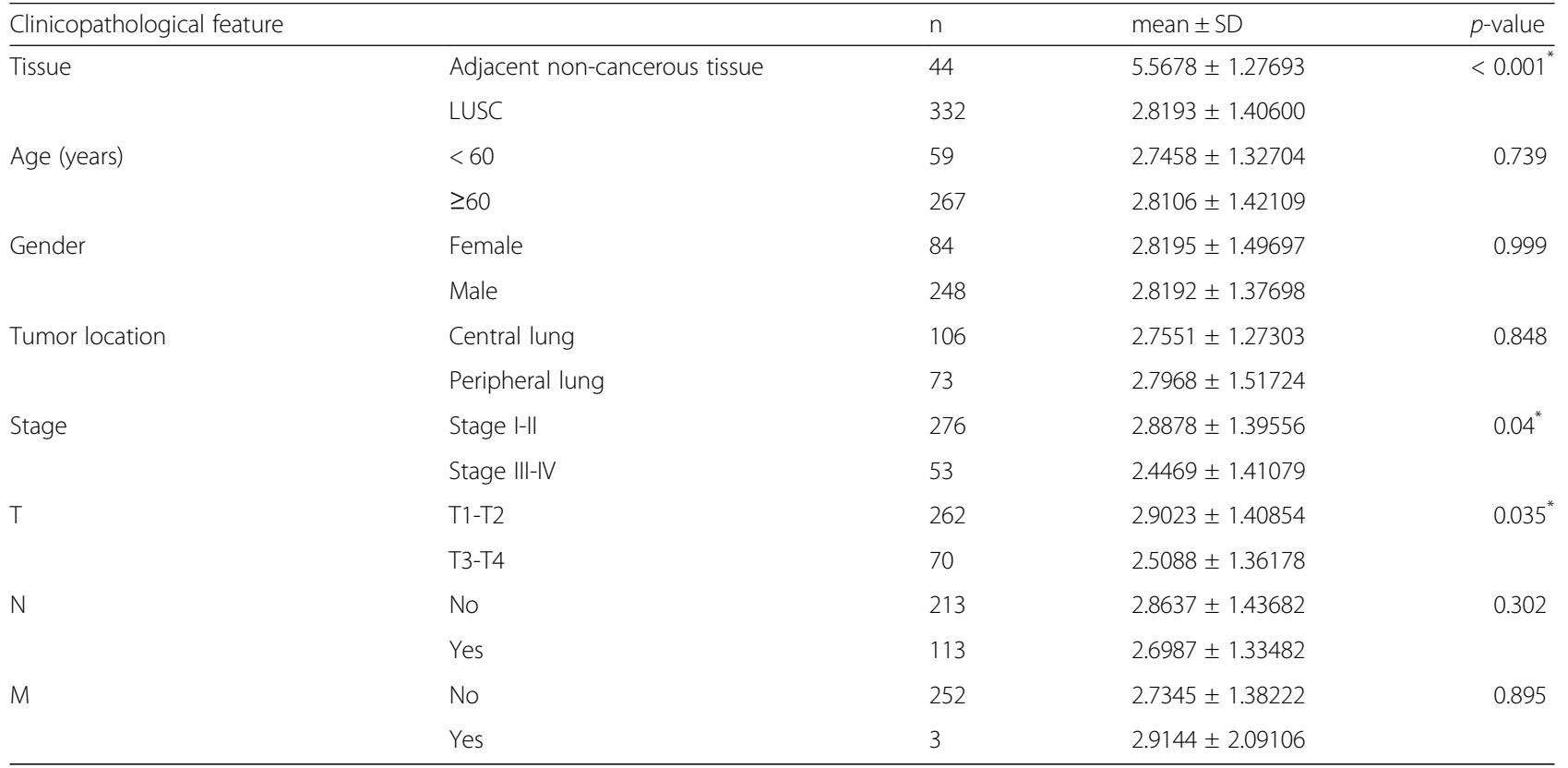

SD Standard deviation, LUSC Lung squamous cell carcinoma

${ }^{*} p<0.05$ was considered statistically significant

samples were extracted for integrated meta-analyses with a random effects model because of high heterogeneity $\left(\mathrm{I}^{2}=95.4 \%, p=0.000\right)$. The differences in individuals, NSCLC subtype, and sample source were considered the sources of heterogeneity. As is shown in Fig. 10a, the combined SMD of miR-144-3p was -0.95 with $95 \% \mathrm{CI}$ of $(-1.37,-0.52)$, indicating that less miR-144-3p was expressed in the NSCLC tissue than in the normal tissue. The sensitivity analysis (Fig. 10b) indicated significant differences among the studies; however, no specific study had a significant effect on high heterogeneity. The evaluation of publication bias was performed with Begg's and Egger's tests and a funnel plot (Fig. 10c). In general, the funnel plot was symmetrical, and the $p$ values obtained from the Begg's and Egger's tests were 0.833 and 0.335 , respectively. In sum, the results indicate that publication bias for the studies was controlled passably.

\section{Bioinformatic analyses}

\section{Promising target genes collection}

From miRWALK2.0, 1635 genes targeted by miR144-3p in NSCLC predicted by more than six algorithms were obtained. A total of 1109 overexpressed genes in LUAD were collected on the basis of GEPIA. In addition, 1922 overexpressed genes in LUSC were acquired from GEPIA. After intersection, 34 predicted target genes were selected. Four specific targets of miR-144-3p were verified in previous studies related to
LC (Table 8). The TIGAR is also known as the C12orf5 gene. Accordingly, a total of 37 potential target genes were collected.

\section{Gene ontology and Kyoto encyclopedia of genes and genomes analyses}

For further interpretation of the function of the promising genes targeted by miR-144-3p in NSCLC, KEGG, and $\mathrm{GO}$ annotations were performed in Metascape. For the GO analysis, three categories were used: BP, CC, and MF. For the BP, renal system development (GO: 0072001) and the nucleobase-containing small molecule metabolic process (GO: 0055086) were the top two pathways (Fig. 11a). For the CC, the potential target differentially expressed genes (DEGs) were predominantly enriched in the centriole (GO: 0005814), Golgi membrane (GO: 0000139), and mitochondrial envelope (GO: 0005740) (Fig. 11c). For MF, the three significantly involved items were RNA polymerase II proximal promoter sequence-specific DNA binding (GO: 0000978); cofactor binding (GO: 0048037); and transferase activity, transferring glycosyl groups (GO: 0016757) (Fig. 11b). Regarding KEGG, the top two enriched pathways were the protein digestion and absorption (hsa 04974) and the thyroid hormone signaling pathways (hsa04919) (Fig. 12). PPI revealed five genesC12orf5, CEP55, E2F8, STIL, and TOP2A-as hub genes with the threshold value of 6 (Fig. 13). 
Table 3 Association between microRNA-144-3p expression and clinicopathological parameters in lung adenocarcinoma, based on The Cancer Genome Atlas data

\begin{tabular}{lllll}
\hline Clinicopathological & & $\mathrm{n}$ & mean \pm SD & $p$ value \\
\hline Tissue & Adjacent non-cancerous tissue & 43 & $5.2775 \pm 1.64708$ & $2.8959 \pm 1.35967$ \\
& LUAD & 445 & $3.0042 \pm 1.40207$ \\
Age (years) & $<60$ & 120 & $2.7979 \pm 1.33460$ \\
& $\geq 60$ & 306 & $2.9011 \pm 1.33672$ \\
Gender & Female & Male & 239 & $2.8898 \pm 1.38905$ \\
& Central lung & 206 & $2.5665 \pm 1.1857$ \\
Tumor location & Peripheral lung & 54 & $2.747 \pm 1.3564$ \\
& Stage I-II & 113 & $2.8191 \pm 1.33042$ \\
Stage & Stage III-IV & 349 & $3.1868 \pm 1.45395$ \\
& T1-T2 & 91 & $2.9199 \pm 1.35515$ \\
$T$ & T3-T4 & 385 & $2.6359 \pm 1.3106$ \\
$N$ & No & 57 & $2.8546 \pm 1.35977$ \\
$M$ & Yes & 291 & $2.9367 \pm 1.35784$ \\
& No & 145 & $2.8058 \pm 1.31875$ \\
\hline
\end{tabular}

SD Standard deviation, LUAD Lung adenocarcinoma

${ }^{*} p<0.05$ was considered statistically significant

Expression of hub genes from the Cancer genome atlas and the genotype-tissue expression datasets

Of the five hub genes, not including $\mathrm{C} 12$ orf5, that occupied the central region of the PPI network, four genes (CEP55, E2F8, STIL, and TOP2A) were significantly up-regulated in the NSCLC group compared to the control group (Fig. 14).

\section{Discussion}

Although previous studies have documented the expression of miR-144-3p in NSCLC, correlations of the clinical features with NSCLC and miR-144-3p have been seldom reported. This study used a larger number of samples for a systematic investigation of the relationships. A

Table 4 Association between microRNA-144-3p expression and clinicopathological parameters in non-small cell lung cancer, based on The Cancer Genome Atlas data

\begin{tabular}{|c|c|c|c|c|}
\hline Clinicopathological feature & & $n$ & mean $\pm S D$ & $p$-value \\
\hline \multirow[t]{2}{*}{ Tissue } & Adjacent non-cancerous tissue & 87 & $5.4243 \pm 1.4702$ & $<0.001^{*}$ \\
\hline & LUSC+LUAD & 777 & $2.8632 \pm 1.37928$ & \\
\hline \multirow[t]{2}{*}{ Age (years) } & $<60$ & 179 & $2.919 \pm 1.37944$ & 0.330 \\
\hline & $\geq 60$ & 573 & $2.8038 \pm 1.37438$ & \\
\hline \multirow[t]{2}{*}{ Gender } & Female & 323 & $2.8799 \pm 1.37826$ & 0.776 \\
\hline & Male & 454 & $2.8513 \pm 1.38139$ & \\
\hline \multirow[t]{2}{*}{ Tumor location } & Central lung & 160 & $2.6915 \pm 1.24372$ & 0.604 \\
\hline & Peripheral lung & 186 & $2.7666 \pm 1.41787$ & \\
\hline \multirow[t]{2}{*}{ Stage } & Stage I-II & 625 & $2.8494 \pm 1.3589$ & 0.630 \\
\hline & Stage III-IV & 144 & $2.9145 \pm 1.47731$ & \\
\hline \multirow[t]{2}{*}{$\mathrm{T}$} & $\mathrm{T} 1-\mathrm{T} 2$ & 647 & $2.9128 \pm 1.37596$ & $0.008^{*}$ \\
\hline & T3-T4 & 127 & $2.5658 \pm 1.33528$ & \\
\hline \multirow[t]{2}{*}{ N } & No & 504 & $2.8585 \pm 1.39145$ & 0.803 \\
\hline & Yes & 258 & $2.8324 \pm 1.35039$ & \\
\hline \multirow[t]{2}{*}{ M } & No & 535 & $2.7722 \pm 1.34822$ & 0.124 \\
\hline & Yes & 22 & $3.277 \pm 1.45564$ & \\
\hline
\end{tabular}



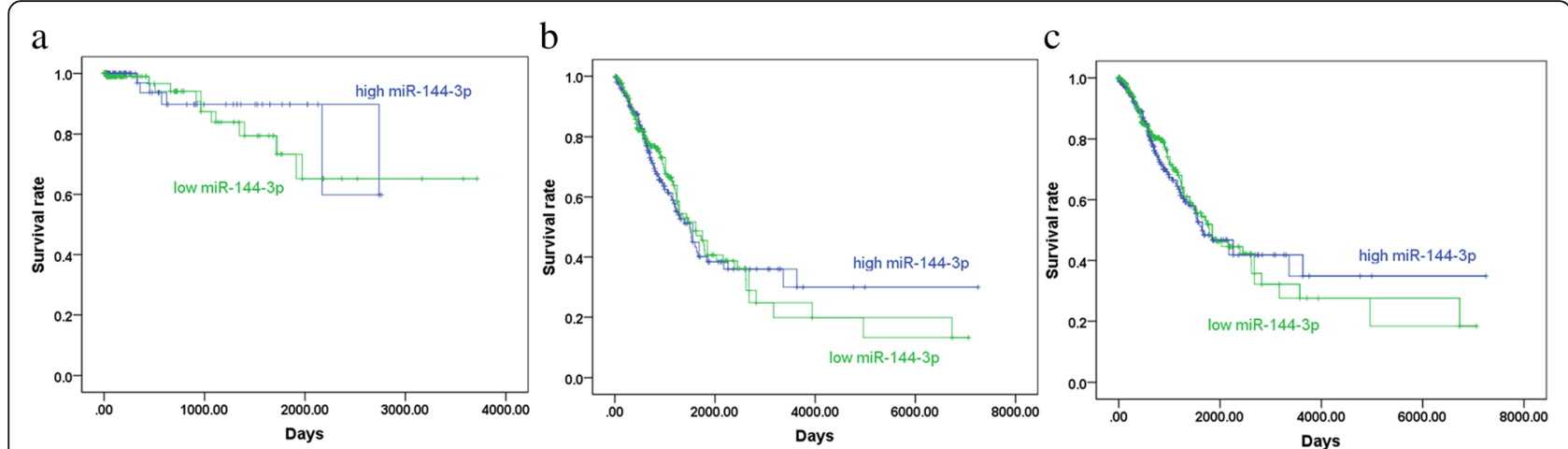

Fig. 7 Kaplan-Meier curves for microRNA-144-3p in a lung squamous cell carcinoma (LUSC), b lung adenocarcinoma (LUAD), and c non-small cell lung cancer (NSCLC) tissues, based on The Cancer Genome Atlas data. Notes: The $p$ values for the survival curves of LUSC, LUAD, and NSCLC were $0.509,0.863$, and 0.808 , respectively. No distinct prognostic differences were observed among them. (green curve: low expression, blue curve: high expression)

highlight of this study was the use of the computational biology method to explore the latent mechanism of miR-144-3p in NSCLC.

A decreased expression of miR-144-3p was found in the NSCLC cases, as presented by the GEO, TCGA, and RT-qPCR. A comprehensive meta-analysis was the focus of the current study. Data were obtained from multiple sources: namely, microarrays, previous studies, RT-qPCR, and TCGA. The meta-analysis results revealed that the decline of miR-144-3p in NSCLC was consistent across studies $[19,22]$. It was therefore concluded that there was a considerable decrease of miR-144-3p expression in NSCLC. Based on the data from TCGA, the miR-144-3p expression level in LUSC and LUAD was related to stage,

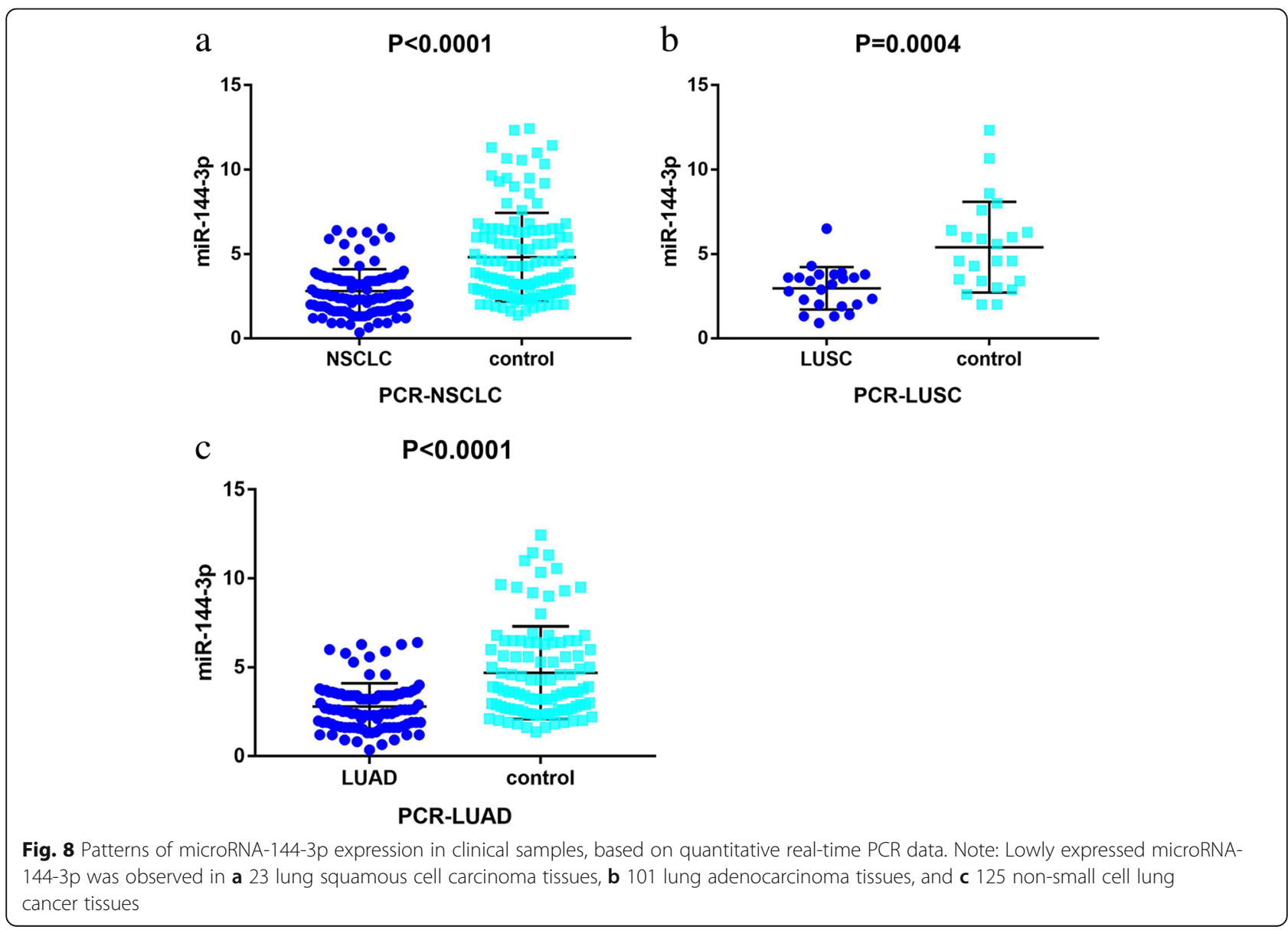


Table 5 Associations between microRNA-144-3p expression and clinicopathological features in non-small cell lung cancer based on quantitative real-time PCR data

\begin{tabular}{|c|c|c|c|c|}
\hline Clinicopathological feature & & $\mathrm{n}$ & mean $\pm S D$ & $p$ value \\
\hline \multirow[t]{2}{*}{ Tissue } & Adjacent non-cancerous tissue & 125 & $4.813 \pm 2.618$ & $<0.001^{*}$ \\
\hline & NSCLC & 125 & $2.808 \pm 1.303$ & \\
\hline \multirow[t]{2}{*}{ Age (years) } & $<60$ & 57 & $2.7879 \pm 1.33904$ & 0.874 \\
\hline & $\geq 60$ & 68 & $2.8254 \pm 1.28196$ & \\
\hline \multirow[t]{2}{*}{ Gender } & Female & 50 & $2.9786 \pm 1.40038$ & 0.247 \\
\hline & Male & 75 & $2.6948 \pm 1.23055$ & \\
\hline \multirow[t]{2}{*}{ Tumor size (cm) } & $\leq 3$ & 60 & $2.9242 \pm 1.44526$ & 0.346 \\
\hline & $>3$ & 65 & $2.7014 \pm 1.15768$ & \\
\hline \multirow[t]{2}{*}{ Smoke } & No & 38 & $3.2489 \pm 1.48252$ & 0.166 \\
\hline & Yes & 30 & $2.7767 \pm 1.29623$ & \\
\hline \multirow[t]{2}{*}{ Lymph node metastasis } & No & 56 & $3.0875 \pm 1.38065$ & $0.033^{*}$ \\
\hline & Yes & 69 & $2.5817 \pm 1.19935$ & \\
\hline \multirow[t]{2}{*}{ Vascular invasion } & No & 90 & $3.0682 \pm 1.24395$ & $<0.001^{*}$ \\
\hline & Yes & 35 & $2.1400 \pm 1.22630$ & \\
\hline \multirow[t]{2}{*}{ TNM } & $|-| \mid$ & 54 & $2.9615 \pm 1.27286$ & 0.251 \\
\hline & III-IV & 71 & $2.6918 \pm 1.32268$ & \\
\hline \multirow[t]{3}{*}{ Histological type } & $A D C$ & 101 & $2.7917 \pm 1.30837$ & 0.561 \\
\hline & SCC & 23 & $2.9643 \pm 1.26398$ & \\
\hline & LCLC & 1 & 0.9000 & \\
\hline
\end{tabular}

SD Standard deviation, NSCLC Non-small cell lung cancer, ADC Adenocarcinoma, SCC Squamous cell carcinoma, LCLC Large-cell lung carcinoma " $p<0.05$ was considered statistically significant

and the predominant difference for $\mathrm{T}$ was found in LUAD only.

The results revealed that miR-144-3p could be involved in the occurrence and development of NSCLC, and low miR-144-3p could indicate the promotion of

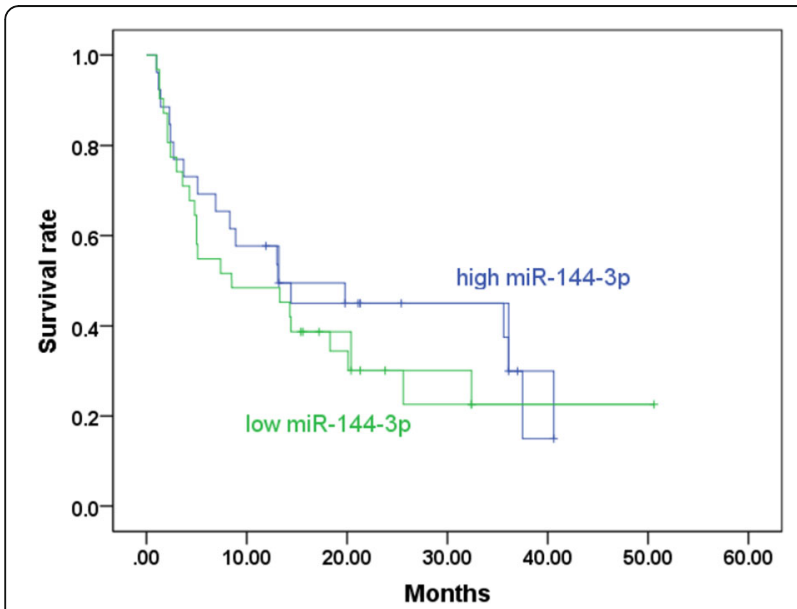

Fig. 9 A Kaplan-Meier curve for microRNA-144-3p in clinical lung adenocarcinoma samples. Note: The $p$ value of the Kaplan-Meier curve for the clinical lung adenocarcinoma patients was 0.397, highlighting a trend of possibly longer overall survival rates in the high microRNA-144$3 p$ group. (green curve: low expression, blue curve: high expression)
NSCLC. Regarding RT-qPCR, miR-144-3p expression was related to lymph node metastasis and vascular invasion. Moreover, the patients with lymph node metastasis and vascular invasion had a tendency to down-regulate miR-144-3p expression. Regarding LUAD, the amount of miR-144-3p differed greatly depending on the smoking status of the patient, the existence of lymph node metastasis, and the presence of vascular invasion. However, no statistical differences were found for LUSC. In sum, miR-144-3p might serve as a marker to monitor the progression of NSCLC.

No differences were observed in the survival times for the low-miR-144-3p group and the high-miR144-3p group, according to TCGA data. In contrast, the PCR results revealed a trend that suggests that LUAD patients with lower miR-144-3p levels might have worse outcomes although not to a significant extent. A study by $\mathrm{Wu}$ et al. [23] revealed that miR-144-3p was one of the independent prognostic risk factors for LUAD patients, with those with low miR-144-3p having poor prognoses. Further investigations are required for corroboration.

At the present time, the specific molecular mechanism of NSCLC is not widely understood. Therefore, bioinformatics analyses were performed to discover the inherent mode of NSCLC activity at the molecular level. Based 
Table 6 Associations between microRNA-144-3p expression and clinicopathological features in lung squamous cell carcinoma, based on quantitative real-time PCR data

\begin{tabular}{|c|c|c|c|c|}
\hline Clinicopathological feature & & $n$ & mean $\pm S D$ & $p$ value \\
\hline \multirow[t]{2}{*}{ Tissue } & Adjacent non-cancerous tissue & 23 & $5.405 \pm 2.684$ & $0.0004^{*}$ \\
\hline & LUSC & 23 & $2.964 \pm 1.264$ & \\
\hline \multirow[t]{2}{*}{ Age (years) } & $<60$ & 15 & $2.872 \pm 1.39748$ & 0.611 \\
\hline & $\geq 60$ & 8 & $3.1375 \pm 1.03086$ & \\
\hline \multirow[t]{2}{*}{ Gender } & Female & 5 & $2.976 \pm 0.6827$ & 0.974 \\
\hline & Male & 18 & $2.9611 \pm 1.39922$ & \\
\hline \multirow[t]{2}{*}{ Tumor size $(\mathrm{cm})$} & $\leq 3$ & 7 & $3.02 \pm 1.79763$ & 0.915 \\
\hline & $>3$ & 16 & $2.94 \pm 1.02398$ & \\
\hline \multirow[t]{2}{*}{ Smoke } & No & 12 & $2.6733 \pm 1.1468$ & 0.263 \\
\hline & Yes & 11 & $3.2818 \pm 1.36222$ & \\
\hline \multirow[t]{2}{*}{ Lymph node metastasis } & No & 11 & $3.0436 \pm 1.48816$ & 0.784 \\
\hline & Yes & 12 & $2.8917 \pm 1.08163$ & \\
\hline \multirow[t]{2}{*}{ Vascular invasion } & No & 20 & $2.919 \pm 0.99752$ & 0.667 \\
\hline & Yes & 3 & $3.2667 \pm 2.82194$ & \\
\hline \multirow[t]{2}{*}{ TNM } & $|-| \mid$ & 10 & $2.978 \pm 0.89643$ & 0.963 \\
\hline & III-IV & 13 & $2.9538 \pm 1.52513$ & \\
\hline
\end{tabular}

SD Standard deviation, LUSC Lung squamous cell carcinoma

${ }^{*} p<0.05$ was considered statistically significant

on miRWALK2.0 and TCGA data, the candidate targets of miR-144-3p were projected. In an attempt to explore the roles of these genes further, KEGG and GO annotation analyses were performed. According to GO enrichment, the candidate targets of miR-144-3p might have an important effect on the progression of NSCLC by modulating several cellular biology processes, such as renal system development. Moreover, these genes could also have an important effect, such as cofactor binding, on MF. The results of the KEGG analysis also showed

Table 7 Associations between microRNA-144-3p expression and clinicopathological features in lung adenocarcinoma, based on quantitative real-time PCR data

\begin{tabular}{|c|c|c|c|c|}
\hline Clinicopathological feature & & $n$ & mean $\pm S D$ & $p$-value \\
\hline \multirow[t]{2}{*}{ Tissue } & Adjacent non-cancerous tissue & 101 & $4.693 \pm 2.607$ & $<0.001^{*}$ \\
\hline & LUAD & 101 & $2.792 \pm 1.308$ & \\
\hline \multirow[t]{2}{*}{ Age (years) } & $<60$ & 41 & $2.8032 \pm 1.31708$ & 0.942 \\
\hline & $\geq 60$ & 60 & $2.7838 \pm 1.31347$ & \\
\hline \multirow[t]{2}{*}{ Gender } & Female & 45 & $2.9789 \pm 1.46340$ & 0.211 \\
\hline & Male & 56 & $2.6413 \pm 1.16082$ & \\
\hline \multirow[t]{2}{*}{ Tumor size $(\mathrm{cm})$} & $\leq 3$ & 53 & $2.9115 \pm 1.41270$ & 0.332 \\
\hline & $>3$ & 48 & $2.6594 \pm 1.18327$ & \\
\hline \multirow[t]{2}{*}{ Smoke } & No & 26 & $3.5146 \pm 1.56261$ & $0.027^{*}$ \\
\hline & Yes & 18 & $2.5722 \pm 1.16539$ & \\
\hline \multirow[t]{2}{*}{ Lymph node metastasis } & No & 45 & $3.0982 \pm 1.3707$ & $0.037^{*}$ \\
\hline & Yes & 56 & $2.5454 \pm 1.21274$ & \\
\hline \multirow[t]{2}{*}{ Vascular invasion } & No & 70 & $3.1109 \pm 1.30906$ & $<0.001^{*}$ \\
\hline & Yes & 31 & $2.0710 \pm 0.99515$ & \\
\hline \multirow[t]{2}{*}{ TNM } & $|-| \mid$ & 44 & $2.9577 \pm 1.35229$ & 0.269 \\
\hline & III-IV & 57 & $2.6635 \pm 1.27056$ & \\
\hline
\end{tabular}




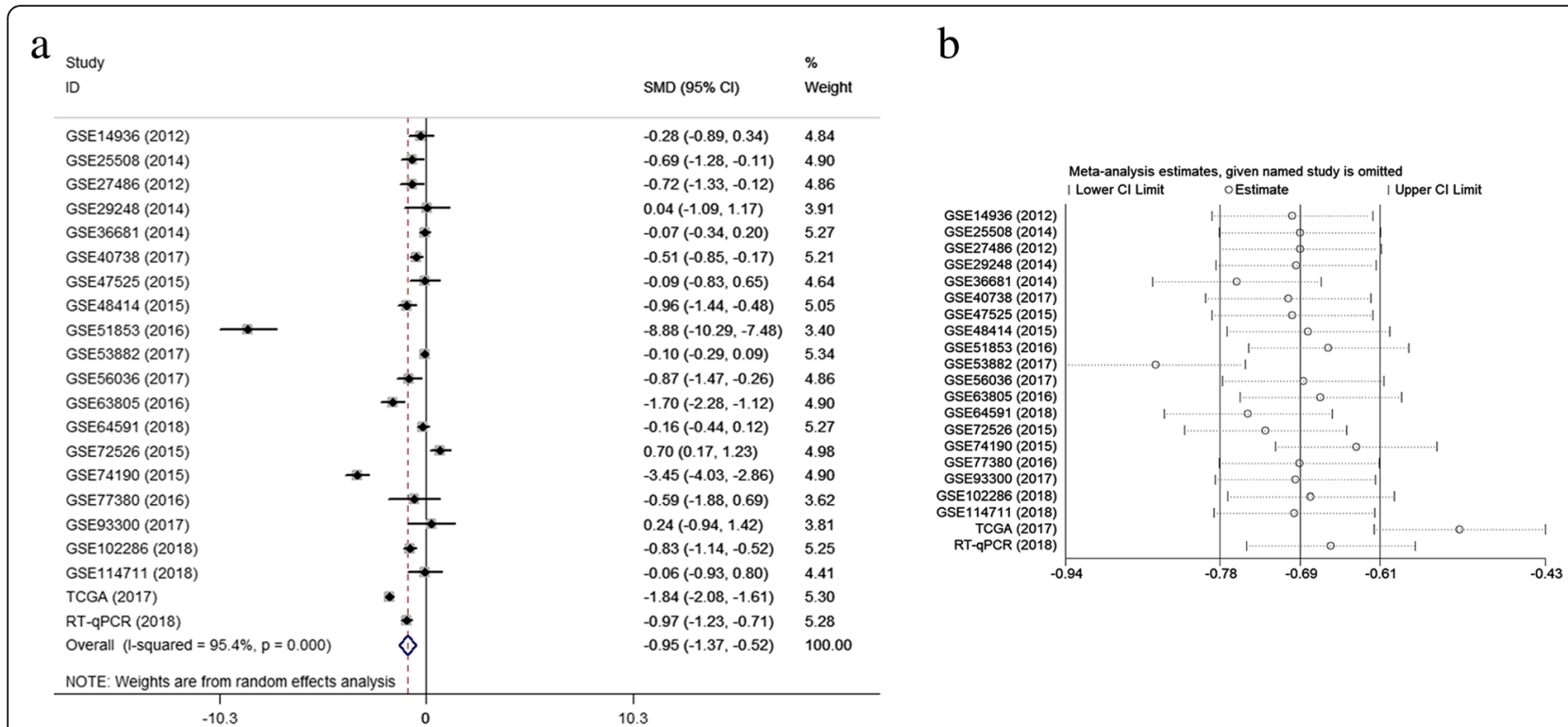

C

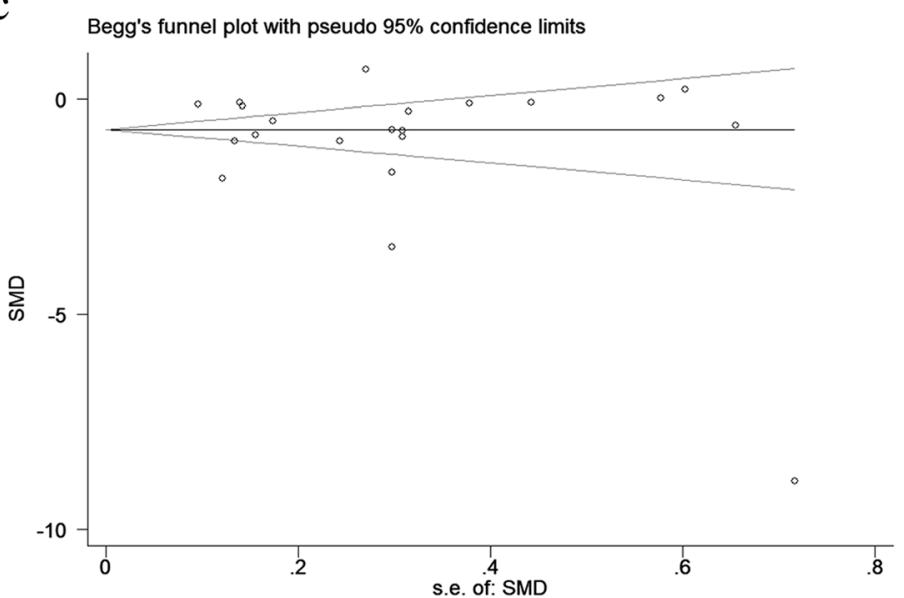

Fig. 10 A comprehensive meta-analysis based on Gene Expression Omnibus (GEO), The Cancer Genome Atlas (TCGA), and quantitative real-time PCR (RT-qPCR) datasets. a Forest plot of microRNA-144-3p expression data from GEO, TCGA, and RT-qPCR datasets. With the random effects model, the $I^{2}$ value was $95.4 \%$. b Sensitivity analysis of GEO, TCGA, and RT-qPCR datasets. $\mathbf{c}$ The evaluation of the publication bias of the GEO, TCGA, and RT-qPCR datasets (Begg's test, $p=0.833$ )

the roles of the candidate targets of miR-144-3p in NSCLC. The top two enriched pathways were the protein digestion and absorption and the thyroid hormone signaling pathways. This suggests that the promising targets of miR-144-3p could be involved in the

Table $\mathbf{8}$ Identified target genes derived from the literature

\begin{tabular}{ll}
\hline validated target gene & PMID \\
\hline BLACAT1 & PMID: 28885863 \\
GLUT1 & PMID: 27313692 \\
TIGAR & PMID: 25660220 \\
ZEB1 & PMID: 26191328 \\
\hline
\end{tabular}

aforementioned pathways to influence the occurrence and progression of NSCLC.

The protein digestion and absorption pathway is a key pathway in several human cancers. A study by Shi et al. showed that this pathway might contribute to the pulmonary metastasis of osteosarcoma patients [24]. It might be involved in the up-regulation of differentially expressed genes in breast cancer [25], and it has already been associated with the down-regulated differentially expressed genes in pancreatic neuroendocrine tumors [26]. The protein digestion and absorption pathway is connected mainly to differentially expressed genes, and this affects the occurrence and development of enchondromas [27]. Additionally, B-cell malignancies are relevant 
a

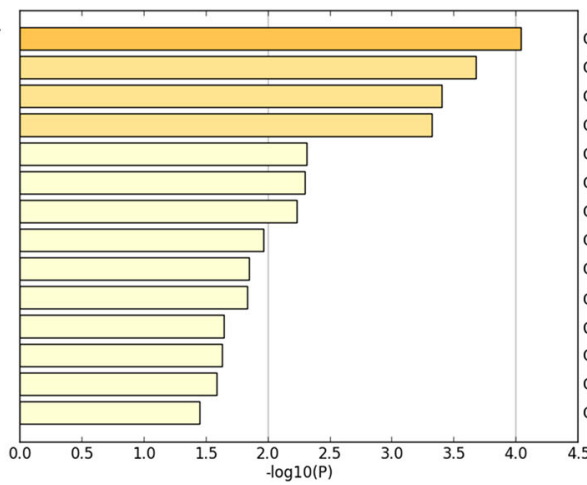

b

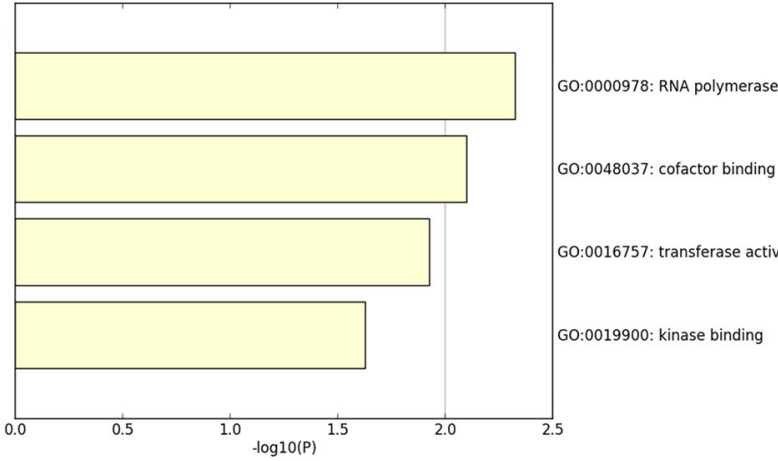

$\mathrm{C}$

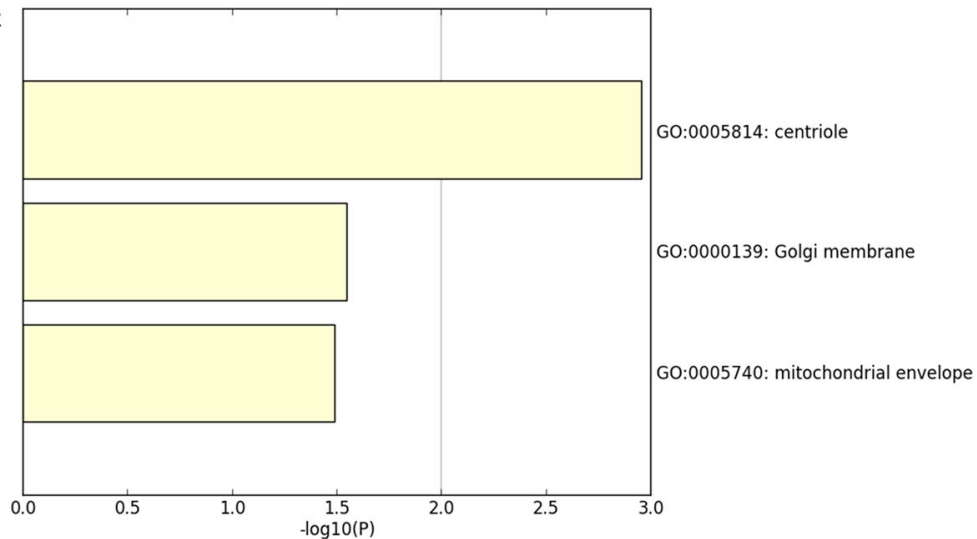

60:0072001: renal system development

0:0005975: carbohydrate metabolic process

GO:0048704: embryonic skeletal system morphogenesis

GO:0009612: response to mechanical stimulus

1903530: regulation of secretion by cell

GO:0009100: glycoprotein metabolic process

GO:1901214: regulation of neuron death

0009615: response to virus

GO:0051301: cell division

GO:0001503: ossification

GO:0034504: protein localization to nucleus

GO:0051052: regulation of DNA metabolic process

GO:0051656: establishment of organelle localization

$$
\text { (1) }
$$

GO:0055086: nucleobase-containing small molecule metabolic process 


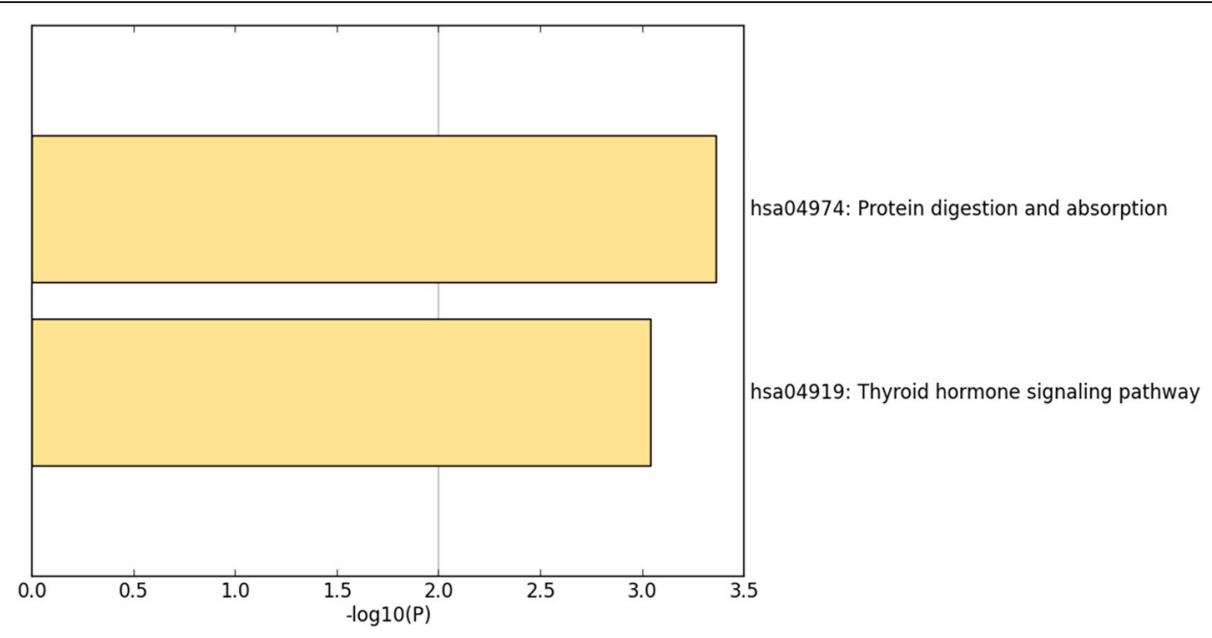

Fig. 12 Distribution of Kyoto Encyclopedia of Genes and Genomes terms for the target genes of microRNA-144-3p in non-small cell lung cancer. Note: The protein digestion and absorption and the thyroid hormone signaling pathways were the top two pathways most strongly enriched by the target genes

The current study has limitations. Because a large-scale clinical sample was not used, more investigations with large clinical samples are required for further confirmation of the function of miR-144-3p in NSCLC prognoses. In addition, experiments were not conducted for the detection of the expression of hub genes. The expression of these genes needs to be verified by additional well-designed studies. Although bioinformatics analyses were performed, the specific molecular mechanisms were not identified.

In conclusion, the current study validated that miR-144-3p was lowly expressed in NSCLC. More importantly, miR-144-3p might function as a latent tumor biomarker in the prognosis prediction for

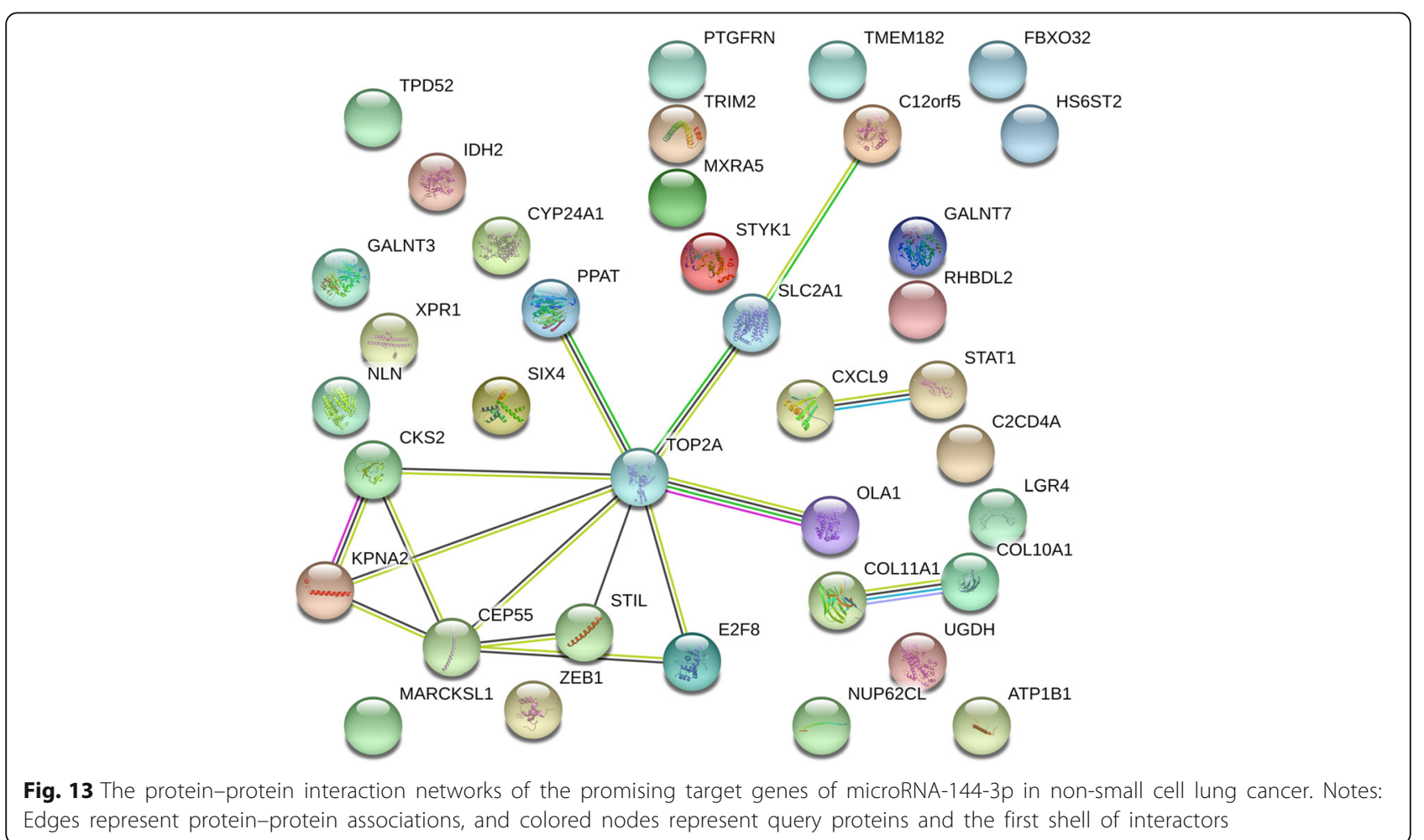


a

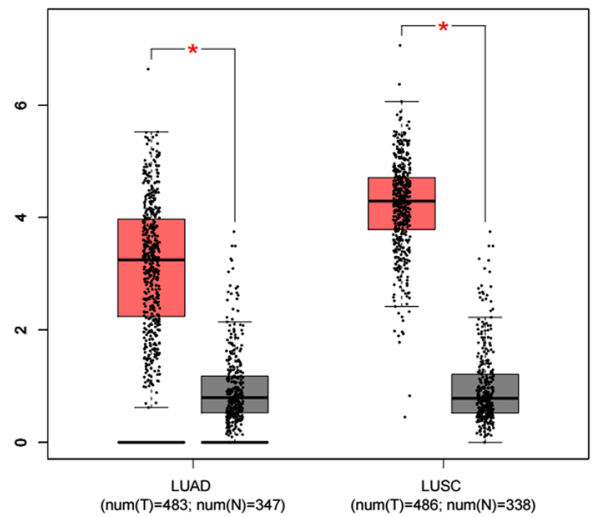

c

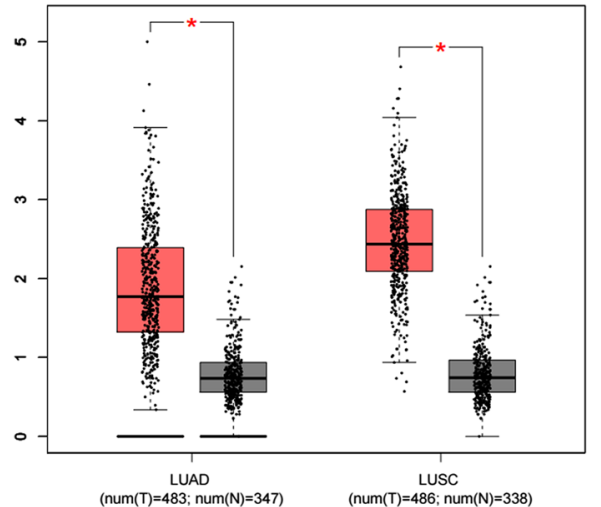

b

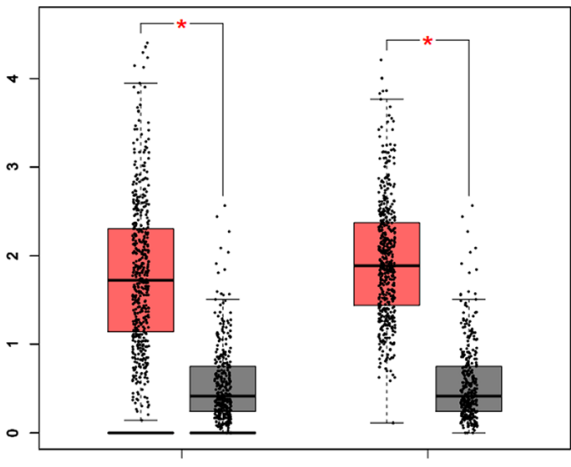

$\operatorname{num}(\mathrm{T})=483 ; \operatorname{num}(\mathrm{N})=347) \quad(\operatorname{num}(\mathrm{T})=486 ; \operatorname{num}(\mathrm{N})=338)$

d

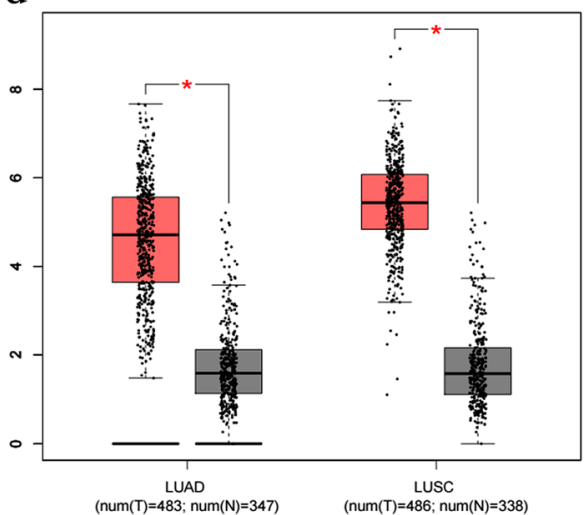

Fig. 14 Expression of hub genes in non-small cell lung cancer and normal tissues, based on Gene Expression Profiling Interactive Analysis (GEPI A). Notes: Expression of the hub genes was detected in 969 NSCLC tissues (T) and 685 normal tissues (N) on the basis of GEPIA. Four of the genes-a CEP55, b E2F8, c STIL, and $\mathbf{d}$ TOP2A-were overexpressed in non-small cell lung cancer tissues when compared to the normal tissues

NSCLC. The results of bioinformatics analyses may present a new method for investigating the pathogenesis of NSCLC.

\section{Abbreviations}

BP: Biological process; CC: Cellular component; Cl: Confidential interval; GEO: Gene Expression Omnibus; GO: Gene Ontology; KEGG: Kyoto Encyclopedia of Genes and Genomes; LC: Lung cancer; LCC: Lung large cell carcinoma; LUAD: Lung adenocarcinoma; LUSC: Lung squamous cell carcinoma; M: Mean; MF: Molecular function; NSCLC: Non-small cell lung cancer; PPI: Protein-protein interaction network; qRT-PCR: Quantitative realtime PCR; SD: Standard deviation; SMD: Standardized mean difference; TCGA: The Cancer Genome Atlas

\section{Acknowledgments}

None.

\section{Funding}

The current study was supported by the future academic star project of Guangxi Medical University (2017).

\section{Availability of data and materials}

One hundred twenty-five (125) paired non-small cell lung cancer specimens were obtained from the First Affiliated Hospital of Guangxi Medical University (Guangxi, China).

\section{Authors' contributions}

TQG and GC conceived and designed the study. YJC, WQX, and HMH drafted the manuscript. YJC, HMH, KS and SPH participated in the data collection, analysis and interpretation, and statistical analysis. YJC, YNG and WQX revised the manuscript. TQG, GC, KLW and ZYL were responsible for quality control. All of the authors have read and approved the manuscript.

Ethics approval and consent to participate

This study was approved by the Ethics Committee of the First Affiliated Hospital of Guangxi Medical University.

\section{Consent for publication}

Not applicable.

\section{Competing interests}

The authors declare that they have no competing interests.

\section{Publisher's Note}

Springer Nature remains neutral with regard to jurisdictional claims in published maps and institutional affiliations.

\section{Author details}

'Department of Medical Oncology, Second Affiliated Hospital of Guangxi Medical University, Daxuedong Road, Nanning, Guangxi Zhuang Autonomous Region 530021, People's Republic of China. ${ }^{2}$ Department of Pathology, Second Affiliated Hospital of Guangxi Medical University, Daxuedong Road, Nanning, Guangxi Zhuang Autonomous Region 530021, People's Republic of China. ${ }^{3}$ Department of Pathology, First Affiliated 
Hospital of Guangxi Medical University, Shuangrong Road, Nanning, Guangxi Zhuang Autonomous Region 530021, People's Republic of China.

Received: 17 September 2018 Accepted: 31 January 2019 Published online: 04 March 2019

\section{References}

1. Siegel RL, Miller KD, Jemal A. Cancer statistics, 2018. CA Cancer J Clin. 2018; 68:7-30.

2. Inamura K. Lung cancer: understanding its molecular pathology and the 2015 who classification. Front Oncol. 2017:7:193.

3. Hirsch FR, Scagliotti GV, Mulshine JL, Kwon R, Curran WJ Jr, Wu YL, et al. Lung cancer: current therapies and new targeted treatments. Lancet. 2017; 389:299-311.

4. Yun YH, Kim YA, Sim JA, Shin AS, Chang YJ, Lee J, et al. Prognostic value of quality of life score in disease-free survivors of surgically-treated lung cancer. BMC Cancer. 2016;16:505

5. Tang Y, Qiao G, Xu E, Xuan Y, Liao M, Yin G. Biomarkers for early diagnosis, prognosis, prediction, and recurrence monitoring of non-small cell lung cancer. Onco Targets Ther. 2017;10:4527-34.

6. Rios J, Gosain R, Goulart BH, Huang B, Oechsli MN, McDowell JK, et al. Treatment and outcomes of non-small-cell lung cancer patients with high comorbidity. Cancer Manag Res. 2018;10:167-75.

7. Beermann J, Piccoli MT, Viereck J, Thum T. Non-coding RNAs in development and disease: background, mechanisms, and therapeutic approaches. Physiol Revs. 2016:96:1297.

8. Bracken CP, Scott HS, Goodall GJ. A network-biology perspective of microRNA function and dysfunction in cancer. Nat Rev Genet. 2016:17:719.

9. Zhou Q, Huang SX, Zhang F, Li SJ, Liu C, Xi YY, et al. MicroRNAs: a novel potential biomarker for diagnosis and therapy in patients with non-small cell lung cancer. Cell Prolif. 2017. https://doi.org/10.1111/cpr.12394.

10. Kang L, Tao X, He BS, Pan YQ, Sun HL, Peng HX, et al. MicroRNA expression profiles predict progression and clinical outcome in lung adenocarcinoma. Onco Targets Ther. 2016;9:5679-92.

11. Zhang SY, Lu ZM, Lin YF, Chen LS, Luo XN, Song XH, et al. miR-144-3p, a tumor suppressive microRNA targeting ETS-1 in laryngeal squamous cell carcinoma. Oncotarget. 2016;7:11637.

12. Li B, Zhang S, Hao S, Li C. MicroRNA-144-3p suppresses gastric cancer progression by inhibiting epithelial-to-mesenchymal transition through targeting PBX3. Biochem Biophys Res Commun. 2017;484:241-7.

13. Liang $H$, Ye Z, Yin S, Mo W, Wang H, Zhao J, et al. A comprehensive insight into the clinicopathologic significance of miR-144-3p in hepatocellular carcinoma. Onco Targets Ther. 2017;10:3405-19.

14. Li J, Sun P, Yue Z, Zhang D, You K, Wang J. miR-144-3p induces cell cycle arrest and apoptosis in pancreatic cancer cells by targeting proline-rich protein 11 expression via the mitogen-activated protein kinase signaling pathway. DNA Cell Biol. 2017;36:619.

15. Xiao W, Lou N, Ruan H, Bao L, Xiong Z, Yuan C, et al. Mir-144-3p promotes cell proliferation, metastasis, sunitinib resistance in clear cell renal cell carcinoma by downregulating ARID1A. Cell Physiol Biochem. 2017:43:2420-33.

16. Zhang LY, Hofun LV, Wong AM, Kwong DL, Zhu YH, Dong SS, et al. MicroRNA-144 promotes cell proliferation, migration and invasion in nasopharyngeal carcinoma through repression of PTEN. Carcinogenesis. 2013:34:454

17. Ye JJ, Cao J. MicroRNAs in colorectal cancer as markers and targets: recent advances. World J Gastroenterol. 2014;20:4288.

18. Chen S, Li P, Li J, Wang Y, Du Y, Chen X, et al. MiR-144 inhibits proliferation and induces apoptosis and autophagy in lung cancer cells by targeting TIGAR. Cell Physiol Biochem. 2015;35:997-1007.

19. Liu M, Gao J, Huang Q, Jin Y, Wei Z. Downregulating microRNA-144 mediates a metabolic shift in lung cancer cells by regulating GLUT1 expression. Oncol Lett. 2016;11:3772.

20. Dweep H, Gretz N. miRWalk2.0: a comprehensive atlas of microRNA-target interactions. Nat Methods. 2015;12:697.

21. Szklarczyk D, Franceschini A, Wyder S, Forslund K, Heller D, Huerta-Cepas J, et al. STRING v10: protein-protein interaction networks, integrated over the tree of life. Nucleic Acids Res. 2015:43:D447-52.

22. Zhang G, An H, Fang X. MicroRNA-144 regulates proliferation, invasion, and apoptosis of cells in malignant solitary pulmonary nodule via zinc finger $\mathrm{E}$ box-binding homeobox 1. Int J Clin Exp Pathol. 2015;8:5960-7.
23. Wu C, Xu B, Zhou Y, Ji M, Zhang D, Jiang J, Wu C. Correlation between serum IL-1 $\beta$ and miR-144-3p as well as their prognostic values in LUAD and LUSC patients. Oncotarget. 2016;7:85876-87.

24. Shi Z, Zhou H, Pan B, Lu L, Wei Z, Shi L, et al. Exploring the key genes and pathways of osteosarcoma with pulmonary metastasis using a gene expression microarray. Mol Med Rep. 2017;16:7423-31.

25. Dong LF, Xu SY, Long JP, Wan F, Chen YD. RNA-sequence analysis reveals differentially expressed genes (DEGs) in patients exhibiting different risks of tumor metastasis. Med Sci Monit. 2017:23:2842-9.

26. Wang DD, Liu ZW, Han MM, Zhu ZM, Tu YL, Dou CQ, et al. Microarray based analysis of gene expression patterns in pancreatic neuroendocrine tumors. Eur Rev Med Pharmacol Sci. 2015;19:3367-74.

27. Shi Z, Zhou H, Pan B, Lu L, Kang Y, Liu L, et al. Exploring the key genes and pathways in enchondromas using a gene expression microarray. Oncotarget. 2017:8:43967-77.

28. Hsu LI, Briggs F, Shao X, Metayer C, Wiemels JL, Chokkalingam AP, et al. Pathway analysis of genome-wide association study in childhood leukemia among Hispanics. Cancer Epidemiol Biomark Prev. 2016;25:815-22.

29. Ma XP, Zhang W, Wu BQ, Qin J. Correlations between mRNA levels of Centrosomal protein 55 (CEP55) and clinical features of patients with lung cancer. Med Sci Monit. 2018;24:3093-7.

30. Liu L, Mei Q, Zhao J, Dai Y, Fu Q. Suppression of CEP55 reduces cell viability and inducesapoptosis in human lung cancer. Oncol Rep. 2016;36:1939-45.

31. Sun J, Shi R, Zhao S, Li X, Lu S, Bu H, et al. E2F8, a direct target of miR-144, promotes papillary thyroid cancer progression via regulating cell cycle. Exp Clin Cancer Res. 2017:36:40
Ready to submit your research? Choose BMC and benefit from:

- fast, convenient online submission

- thorough peer review by experienced researchers in your field

- rapid publication on acceptance

- support for research data, including large and complex data types

- gold Open Access which fosters wider collaboration and increased citations

- maximum visibility for your research: over $100 \mathrm{M}$ website views per year

At $\mathrm{BMC}$, research is always in progress.

Learn more biomedcentral.com/submissions 